\title{
Convex Relaxation for Optimal Distributed Control Problems
}

\author{
Ghazal Fazelnia, Ramtin Madani, Abdulrahman Kalbat and Javad Lavaei
}

\begin{abstract}
This paper is concerned with the optimal distributed control (ODC) problem for linear discrete-time deterministic and stochastic systems. The objective is to design a static distributed controller with a pre-specified structure that is globally optimal with respect to a quadratic cost functional. It is shown that this NP-hard problem has a quadratic formulation, which can be relaxed to a semidefinite program (SDP). If the SDP relaxation has a rank-1 solution, a globally optimal distributed controller can be recovered from this solution. By utilizing the notion of treewidth, it is proved that the nonlinearity of the ODC problem appears in such a sparse way that an SDP relaxation of this problem has a matrix solution with rank at most 3 . Since the proposed SDP relaxation is computationally expensive for a largescale system, a computationally-cheap SDP relaxation is also developed with the property that its objective function indirectly penalizes the rank of the SDP solution. Various techniques are proposed to approximate a low-rank SDP solution with a rank-1 matrix, leading to near globally-optimal controllers together with a bound on the optimality degree of each controller. The above results are developed for both finite-horizon and infinite-horizon ODC problems. The SDP relaxations developed in this work are exact for the design of a centralized controller, hence serving as an alternative for solving Riccati equations. The efficacy of the proposed SDP relaxations is elucidated through a case study on the distributed frequency control of power systems.
\end{abstract}

\section{INTRODUCTION}

The area of decentralized control is created to address the challenges arising in the control of real-world systems with many interconnected subsystems. The objective is to design a structurally constrained controller - a set of partially interacting local controllers-with the aim of reducing the computation or communication complexity of the overall controller. The local controllers of a decentralized controller may not be allowed to exchange information. The term distributed control is often used in lieu of decentralized control in the case where there is some information exchange between the local controllers (possibly distributed over a geographical area). It has been long known that the design of a globally optimal decentralized (distributed) controller is a daunting task because it amounts to an NP-hard optimization problem in general [1], [2]. Great effort has been devoted to investigating this highly complex problem for special types of systems, including spatially distributed systems [3]-[7], dynamically decoupled systems [8], [9], weakly coupled systems [10], and strongly connected systems [11]. Another special case that has received considerable attention is the design of an optimal static distributed controller [12], [13]. Early approaches

Ghazal Fazelnia is with the Electrical Engineering Department, Columbia University (gf2293@columbia.edu). Abdulrahman Kalbat is with the Electrical Engineering Department, United Arab Emirates University (akalbat@uaeu.ac.ae). Ramtin Madani and Javad Lavaei are with the Department of Industrial Engineering and Operations Research, University of California, Berkeley (ramtin.madani@berkeley.edu and lavaei@berkeley.edu). This work was supported by ONR YIP Award, DARPA Young Faculty Award, NSF CAREER Award 1351279, and NSF EECS Awards 1406894. for the optimal decentralized control problem were based on parameterization techniques [14], [15], which were then evolved into matrix optimization methods [16], [17]. In fact, with a structural assumption on the exchange of information between subsystems, the performance offered by linear static controllers may be far less than the optimal performance achievable using a nonlinear time-varying controller [1].

Due to the recent advances in the area of convex optimization, the focus of the existing research efforts has shifted from deriving a closed-form solution for the above control synthesis problem to finding a convex formulation of the problem that can be efficiently solved numerically [18]-[21]. This has been carried out in the seminal work [22] by deriving a sufficient condition named quadratic invariance, which has been specialized in [23] by deploying the concept of partially order sets. These conditions have been further investigated in several other papers [24]-[26]. A different approach is taken in the recent papers [27] and [28], where it has been shown that the distributed control problem can be cast as a convex optimization for positive systems. The recent literature witnesses that the design of a fully decentralized controller is much more complicated than the design of a distributed controller with a sufficient number of interactions among the local controllers.

Semidefinite programming (SDP) relaxation usually converts an optimization problem with a vector variable to a convex program with a matrix variable, via a lifting technique. The exactness of the relaxation can then be interpreted as the existence of a low-rank (e.g., rank-1) solution for the SDP relaxation. Several papers have studied the existence of a lowrank solution to matrix optimization problems with linear or nonlinear constraints. For instance, the papers [29] and [30] provide upper bounds on the lowest rank among all solutions of a feasible linear matrix inequality problem. A rank-1 matrix decomposition technique is developed in [31] to find a rank-1 solution whenever the number of constraints is small. We have shown in [32] and [33] that semidefinite relaxation is able to solve a large class of non-convex energy-related optimization problems performed over power networks. We related the success of the relaxation to the hidden structure of such problems induced by the physics of a power grid. Inspired by this positive result, we developed the notion of "nonlinear optimization over graph" in [34]-[36]. Our technique maps the structure of an abstract nonlinear optimization into a graph from which the exactness of SDP relaxation may be concluded. By adopting the graph technique developed in [34], the objective of the present work is to study the potential of SDP relaxation for the optimal distributed control problem.

In this paper, we cast the optimal distributed control (ODC) problem as a non-convex optimization problem with only quadratic scalar and matrix constraints, from which an SDP 
relaxation can be obtained. The goal is to show that this relaxation has a low-rank solution whose rank depends on the topology of the controller to be designed. In particular, we prove that the design of a static distributed controller with a pre-specified structure amounts to a sparse SDP relaxation with a solution of rank at most 3 . This positive result is a consequence of the fact that the sparsity graph associated with the underlying optimization problem has a small treewidth. The notion of "treewidth" used in this paper could potentially help to understand how much approximation is needed to make the ODC problem tractable. This is due to a recent result stating that a rank-constrained optimization problem has an almost equivalent convex formulation whose size depends on the treewidth of a certain graph [37]. In this work, we also discuss how to round the rank-3 SDP matrix to a rank-1 matrix in order to design a near globally-optimal controller.

We adopt two different formulations to study the finiteand infinite-horizon distributed control problems. In the finitehorizon case, the state vectors, controller inputs and measured outputs at all times over the horizon are considered as decision variables. This leads to a static optimization problem with time-domain variables, which is regarded as the "time-domain formulation". In the infinite-horizon case, the system dynamics are imposed through a Lyapunov equation as opposed to the time-domain system equations. In this case, we cast the ODC problem as an optimization problem with respect to a Lyapunov matrix instead of time-domain variables, which is referred to as the "Lyapunov-domain formulation". The results of this work hold true for both the time-domain formulation corresponding to the finite-horizon control problem and the Lyapunov-domain formulation associated with the infinitehorizon deterministic/stochastic control problem.

We first investigate the ODC problem for the deterministic systems and then the ODC problem for stochastic systems. Our approach rests on formulating each of these problems as a rank-constrained optimization from which an SDP relaxation can be derived. Since the proposed relaxations with guaranteed low-rank solutions are computationally expensive, we also design computationally-cheap SDP relaxations for numerical purposes. Afterwards, we develop some heuristic methods to recover a near-optimal controller from a low-rank SDP solution. Note that the computationally-cheap SDP relaxations associated with the infinite-horizon ODC are exact in both deterministic and stochastic cases for the classical (centralized) LQR and $H_{2}$ problems. We also conduct a case study on the distributed frequency control of electrical power systems using the aforementioned convex relaxation technique. In particular, we perform simulations on a benchmark system for which the designed near-optimal distributed frequency controllers have global optimality degrees above $99 \%$. Although the focus of the paper is on static controllers, it is possible to extend the main approach to accommodate optimization over classes of structured dynamic controllers.

This work is organized as follows. The problem is introduced in Section III and then the SDP relaxation of a quadratically-constrained quadratic program is studied via a graph-theoretic approach. Three different SDP relaxations of the finite-horizon deterministic ODC problem are presented for the static controller design in Section III] The infinite-horizon deterministic ODC problem is studied in Section IV] The results are generalized to an infinite-horizon stochastic ODC problem in Section V Simulations are provided in Section VI followed by concluding remarks in Section VII

\section{A. Notations}

$\mathbb{R}, \mathbb{S}_{n}$ and $\mathbb{S}_{n}^{+}$denote the sets of real numbers, $n \times n$ symmetric matrices and $n \times n$ positive semidefinite matrices, respectively. The $m \times n$ rectangular identity matrix whose $(i, j)$ entry is equal to the Kronecker delta $\delta_{i j}$ is denoted by $I_{m \times n}$ or alternatively $I_{n}$ when $m=n$. The symbols $\operatorname{rank}\{W\}$, $\operatorname{trace}\{W\}$ and null $\{W\}$ denote the rank, trace and null space of a matrix $W$, respectively. The notation $W \succeq 0$ means that $W$ is symmetric and positive semidefinite. Given a matrix $W$, its $(l, m)$ entry is denoted as $W_{l m}$. Given a block matrix $\mathbf{W}$, its $(l, m)$ block is shown as $\mathbf{W}_{l m}$. Given a matrix $M$, its Moore Penrose pseudoinverse is denoted as $M^{+}$. The superscript $(\cdot)^{\text {opt }}$ is used to show a globally optimal value of an optimization parameter. The symbols $(\cdot)^{T}$ and $\|\cdot\|$ denote the transpose and 2-norm operators, respectively. The symbols $\langle\cdot, \cdot\rangle$ and $\|\cdot\|_{F}$ denote the Frobenius inner product and norm of matrices, respectively. The notation $|\cdot|$ shows the size of a vector, the cardinality of a set or the number of vertices a graph, depending on the context. The expected value of a random variable $x$ is shown as $\mathcal{E}\{x\}$. The submatirx of $M$ formed by rows from the set $\mathcal{S}_{1}$ and columns from the set $\mathcal{S}_{2}$ is denoted by $M\left\{\mathcal{S}_{1}, \mathcal{S}_{2}\right\}$. The notation $\mathcal{G}=(\mathcal{V}, \mathcal{E})$ implies that $\mathcal{G}$ is a graph with the vertex set $\mathcal{V}$ and the edge set $\mathcal{E}$.

\section{Preliminaries}

In this paper, the optimal distributed control (ODC) problem is studied based on the following steps:

- First, the problem is cast as a non-convex optimization problem with only quadratic scalar and/or matrix constraints.

- Second, the resulting non-convex problem is formulated as a rank-constrained optimization problem.

- Third, a convex relaxation of the problem is derived by dropping the non-convex rank constraint.

- Last, the rank of the minimum-rank solution of the SDP relaxation is analyzed.

Since there is no unique SDP relaxation for the ODC problem, a major part of this work is devoted to designing a sparse quadratic formulation of the ODC problem with a guaranteed low-rank SDP solution. To achieve this goal, a graph is associated to each SDP, which is then sparsified to contrive a problem with a low-rank solution. Note that this paper significantly improves our recent result in [38].

\section{A. Problem Formulation}

The following variations of the optimal distributed control problem will be studied in this work.

1) Finite-horizon deterministic ODC problem: Consider the discrete-time system

$$
\begin{aligned}
x[\tau+1] & =A x[\tau]+B u[\tau], & & =0,1, \ldots, p-1 \\
y[\tau] & =C x[\tau], & & =0,1, \ldots, p
\end{aligned}
$$




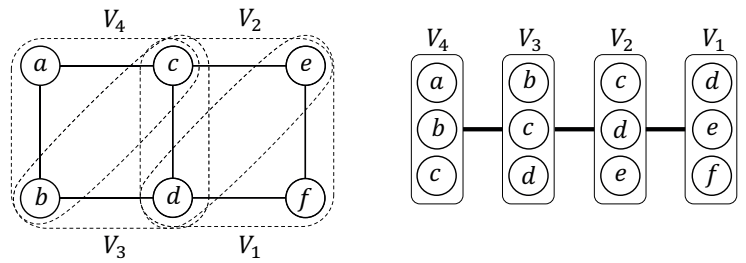

Fig. 1: A minimal tree decomposition for a ladder graph.

with the known matrices $A \in \mathbb{R}^{n \times n}, B \in \mathbb{R}^{n \times m}, C \in \mathbb{R}^{r \times n}$, and $x[0]=c \in \mathbb{R}^{n}$, where $p$ is the terminal time. The goal is to design a distributed static controller $u[\tau]=K y[\tau]$ minimizing a quadratic cost function under the constraint that the controller gain $K$ must belong to a given linear subspace $\mathcal{K} \subseteq \mathbb{R}^{m \times r}$. The set $\mathcal{K}$ captures the sparsity structure of the unknown constrained controller and, more specifically, it contains all $m \times r$ real-valued matrices with forced zeros in certain entries. The cost function

$$
\sum_{\tau=0}^{p}\left(x[\tau]^{T} Q x[\tau]+u[\tau]^{T} R u[\tau]\right)+\alpha\|K\|_{F}^{2}
$$

is considered in this work, where $\alpha$ is a nonnegative scalar, and $Q$ and $R$ are positive-definite matrices. This problem will be studied in Section III.

Remark 1. The third term in the objective function of the ODC problem is a soft penalty term aimed at avoiding a high-gain controller. Instead of this soft penalty, we could impose a hard constraint $\|K\|_{F} \leq \beta$, for a given number $\beta$. The method to be developed later can be adopted for the modified case.

2) Infinite-horizon deterministic ODC problem: The infinite-horizon ODC problem corresponds to the case $p=$ $+\infty$ subject to the additional constraint that the controller must be stabilizing. This problem will be studied through a Lyapunov-domain formulation in Section IV

3) Infinite-horizon stochastic ODC problem: Consider the discrete-time stochastic system

$$
\begin{aligned}
x[\tau+1] & =A x[\tau]+B u[\tau]+E d[\tau], & & \tau=0,1, \ldots \\
y[\tau] & =C x[\tau]+F v[\tau], & & =0,1, \ldots
\end{aligned}
$$

with the known matrices $A, B, C, E$, and $F$, where $d[\tau]$ and $v[\tau]$ denote the input disturbance and measurement noise, which are assumed to be zero-mean white-noise random processes. The ODC problem for the above system will be investigated in Section $\mathrm{V}$.

\section{B. Graph Theory Preliminaries}

Definition 1. For two simple graphs $\mathcal{G}_{1}=\left(\mathcal{V}, \mathcal{E}_{1}\right)$ and $\mathcal{G}_{2}=$ $\left(\mathcal{V}, \mathcal{E}_{2}\right)$ with the same set of vertices, their union is defined as $\mathcal{G}_{1} \cup \mathcal{G}_{2}=\left(\mathcal{V}, \mathcal{E}_{1} \cup \mathcal{E}_{2}\right)$.

Definition 2. The representative graph of an $n \times n$ symmetric matrix $W$, denoted by $\mathcal{G}(W)$, is a simple graph with $n$ vertices whose edges are specified by the locations of the nonzero offdiagonal entries of $W$. In other words, two disparate vertices $i$ and $j$ are connected if $W_{i j}$ is nonzero.

Consider a graph $\mathcal{G}$ identified by a set of "vertices" and a set of edges. This graph may have cycles, in which case it cannot be a tree. Using the notion explained below, we can map $\mathcal{G}$ into a tree $\mathcal{T}$ identified by a set of "nodes" and a set of edges, where each node contains a group of vertices of $\mathcal{G}$.

Definition 3 (Treewidth). Given a graph $\mathcal{G}=(\mathcal{V}, \mathcal{E})$, a tree $\mathcal{T}$ is called a tree decomposition of $\mathcal{G}$ if it satisfies the following properties:

1) Every node of $\mathcal{T}$ corresponds to and is identified by a subset of $\mathcal{V}$.

2) Every vertex of $\mathcal{G}$ is a member of at least one node of $\mathcal{T}$.

3) For every edge $(i, j)$ of $\mathcal{G}$, there should be a node in $\mathcal{T}$ containing vertices $i$ and $j$ simultaneously.

4) Given an arbitrary vertex $k$ of $\mathcal{G}$, the subgraph induced by all nodes of $\mathcal{T}$ containing vertex $k$ must be connected (more precisely, a tree).

Each node of $\mathcal{T}$ is a collection of vertices of $\mathcal{G}$, and is referred to as a bag. The width of $\mathcal{T}$ is the cardinality of its biggest bag minus one. The treewidth of $\mathcal{G}$ is the minimum width over all possible tree decompositions of $\mathcal{G}$ and is denoted by $\operatorname{tw}(\mathcal{G})$.

Every graph has a trivial tree decomposition with one single bag consisting of all vertices of the graph. Figure 1 shows a graph $\mathcal{G}$ with 6 vertices named $a, b, c, d, e, f$, together with its minimal tree decomposition $\mathcal{T}$. Every node of $\mathcal{T}$ is a set containing three members of $\mathcal{V}$. The width of this decomposition is therefore equal to 2 . Observe that the edges of the tree decomposition are chosen in such a way that every subgraph induced by all bags containing each member of $\mathcal{V}$ is a tree (as required by Property 4 stated before).

Note that if $\mathcal{G}$ is a tree itself, it has a minimal tree decomposition $\mathcal{T}$ such that: (i) each bag corresponds to two connected vertices of $\mathcal{G}$, (ii) every two adjacent bags in $\mathcal{T}$ share a vertex in common. Therefore, the treewidth of a tree is equal to 1 . The reader is referred to [39] for a comprehensive literature review on treewidth.

\section{SDP Relaxation}

The objective of this subsection is to study semidefinite programming (SDP) relaxations of a quadratically-constrained quadratic program (QCQP) using a graph-theoretic approach. Consider the standard non-convex QCQP problem

$$
\begin{array}{ll}
\underset{x \in \mathbb{R}^{n}}{\operatorname{minimize}} & f_{0}(x) \\
\text { subject to } & f_{k}(x) \leq 0, \quad k=1, \ldots, q,
\end{array}
$$

where $f_{k}(x)=x^{T} A_{k} x+2 b_{k}^{T} x+c_{k}$ for $k=0, \ldots, q$. Define

$$
F_{k} \triangleq\left[\begin{array}{cc}
c_{k} & b_{k}^{T} \\
b_{k} & A_{k}
\end{array}\right]
$$

Each $f_{k}$ has the linear representation $f_{k}(x)=\left\langle F_{k}, W\right\rangle$ for the following choice of $W$ :

$$
W \triangleq\left[\begin{array}{ll}
x_{0} & x^{T}
\end{array}\right]^{T}\left[\begin{array}{ll}
x_{0} & x^{T}
\end{array}\right]
$$

where $x_{0} \triangleq 1$. On the other hand, an arbitrary matrix $W \in$ $\mathbb{S}_{n+1}$ can be factorized as (6) if and only if it satisfies three properties: $W_{11}=1, W \succeq 0$, and $\operatorname{rank}\{W\}=1$. In this representation of QCQP, the rank constraint carries all the nonconvexity. Neglecting this constraint yields the convex problem 


$$
\begin{array}{cl}
\underset{W \in \mathbb{S}_{n+1}}{\operatorname{minimize}} & \left\langle F_{0}, W\right\rangle \\
\text { subject to } & \left\langle F_{k}, W\right\rangle \leq 0 \quad k=1, \ldots, q, \\
& W_{11}=1, \\
& W \succeq 0,
\end{array}
$$

which is known as an SDP relaxation of the QCQP (4). The existence of a rank-1 solution for an SDP relaxation guarantees the equivalence of the original QCQP and its relaxed problem.

\section{Connection Between Rank and Sparsity}

To explore the rank of the minimum-rank solution of the SDP relaxation problem (7), define $\mathcal{G}=\mathcal{G}\left(F_{0}\right) \cup \cdots \cup \mathcal{G}\left(F_{q}\right)$ as the sparsity graph associated with (7). The graph $\mathcal{G}$ describes the zero-nonzero pattern of the matrices $F_{0}, \ldots, F_{q}$, or alternatively captures the sparsity level of the QCQP problem (4). Let $\mathcal{T}=\left(\mathcal{V}_{\mathcal{T}}, \mathcal{E}_{\mathcal{T}}\right)$ be a tree decomposition of $\mathcal{G}$. Denote its width as $t$ and its bags as $\mathcal{B}_{1}, \mathcal{B}_{2}, \ldots, \mathcal{B}_{|\mathcal{T}|}$. It is known that given such a decomposition, every solution $W^{\text {ref }} \in \mathbb{S}_{n+1}$ of the SDP problem (7) can be transformed into a solution $W^{\text {opt }}$ whose rank is upper bounded by $t+1$ [30]. To perform this transformation, a suitable polynomial-time recursive algorithm will be proposed below.

\section{Matrix completion algorithm:}

1) Set $\mathcal{T}^{\prime}:=\mathcal{T}$ and $W:=W^{\text {ref }}$.

2) If $\mathcal{T}^{\prime}$ has a single node, then consider $W^{\text {opt }}$ as $W$ and terminate; otherwise continue to the next step.

3) Choose a pair of bags of $\mathcal{T}^{\prime}$, namely $\mathcal{B}_{i}$ and $\mathcal{B}_{j}$, such that $\mathcal{B}_{i}$ is a leaf of $\mathcal{T}^{\prime}$ and $\mathcal{B}_{j}$ is its unique neighbor.

4) Using the notation $W\{\cdot, \cdot\}$ introduced in Section I-A let

$$
\begin{aligned}
O & \triangleq W\left\{\mathcal{B}_{i} \cap \mathcal{B}_{j}, \mathcal{B}_{i} \cap \mathcal{B}_{j}\right\} \\
V_{i} & \triangleq W\left\{\mathcal{B}_{i} \backslash \mathcal{B}_{j}, \mathcal{B}_{i} \cap \mathcal{B}_{j}\right\} \\
V_{j} & \triangleq W\left\{\mathcal{B}_{j} \backslash \mathcal{B}_{i}, \mathcal{B}_{i} \cap \mathcal{B}_{j}\right\} \\
H_{i} & \triangleq W\left\{\mathcal{B}_{i} \backslash \mathcal{B}_{j}, \mathcal{B}_{i} \backslash \mathcal{B}_{j}\right\} \in \mathbb{R}^{n_{i} \times n_{i}} \\
H_{j} & \triangleq W\left\{\mathcal{B}_{j} \backslash \mathcal{B}_{i}, \mathcal{B}_{j} \backslash \mathcal{B}_{i}\right\} \in \mathbb{R}^{n_{j} \times n_{j}} \\
S_{i} & \triangleq H_{i}-V_{i} O^{+} V_{i}^{T}=Q_{i} \Lambda_{i} Q_{i}^{T} \\
S_{j} & \triangleq H_{j}-V_{j} O^{+} V_{j}^{T}=Q_{j} \Lambda_{j} Q_{j}^{T}
\end{aligned}
$$

where $Q_{i} \Lambda_{i} Q_{i}^{T}$ and $Q_{j} \Lambda_{j} Q_{j}^{T}$ denote the eigenvalue decompositions of $S_{i}$ and $S_{j}$ with the diagonals of $\Lambda_{i}$ and $\Lambda_{j}$ arranged in descending order. Then, update a part of $W$ as follows:

$W\left\{\mathcal{B}_{j} \backslash \mathcal{B}_{i}, \mathcal{B}_{i} \backslash \mathcal{B}_{j}\right\}:=V_{j} O^{+} V_{i}^{T}+Q_{j} \sqrt{\Lambda_{j}} I_{n_{j} \times n_{i}} \sqrt{\Lambda_{i}} Q_{i}^{T}$

and update $W\left\{\mathcal{B}_{i} \backslash \mathcal{B}_{j}, \mathcal{B}_{j} \backslash \mathcal{B}_{i}\right\}$ accordingly to preserve the Hermitian property of $W$ (note that $\mathcal{B}_{i} \backslash \mathcal{B}_{j}$ is the set-theoretic difference of $\mathcal{B}_{i}$ and $\mathcal{B}_{j}$ ).

5) Update $\mathcal{T}^{\prime}$ by merging $\mathcal{B}_{i}$ into $\mathcal{B}_{j}$, i.e., replace $\mathcal{B}_{j}$ with $\mathcal{B}_{i} \cup \mathcal{B}_{j}$ and then remove $\mathcal{B}_{i}$ from $\mathcal{T}^{\prime}$.

6) Go back to step 2 .

Theorem 1. The output of the matrix completion algorithm, denoted as $W^{\mathrm{opt}}$, is a solution of the SDP problem (7) whose rank is smaller than or equal to $t+1$.
Proof. Consider one run of Step 4 of the matrix completion algorithm. Our first objective is to show that $W\left\{\mathcal{B}_{i} \cup \mathcal{B}_{j}, \mathcal{B}_{i} \cup \mathcal{B}_{j}\right\}$ is a positive semidefinite matrix whose rank is upper bounded by the maximum ranks of $W\left\{\mathcal{B}_{i}, \mathcal{B}_{i}\right\}$ and $W\left\{\mathcal{B}_{j}, \mathcal{B}_{j}\right\}$. To this end, define

$$
S \triangleq\left[\begin{array}{cc}
H_{i} & Z^{T} \\
Z & H_{j}
\end{array}\right]-\left[\begin{array}{c}
V_{i} \\
V_{j}
\end{array}\right] O^{+}\left[\begin{array}{c}
V_{i} \\
V_{j}
\end{array}\right]^{T}=\left[\begin{array}{cc}
Q_{i} & 0 \\
0 & Q_{j}
\end{array}\right] N\left[\begin{array}{cc}
Q_{i}^{T} & 0 \\
0 & Q_{j}^{T}
\end{array}\right]
$$

where $Z \triangleq W\left\{\mathcal{B}_{j} \backslash \mathcal{B}_{i}, \mathcal{B}_{i} \backslash \mathcal{B}_{j}\right\}$ and

$$
N \triangleq\left[\begin{array}{ccc}
\Lambda_{i} & \sqrt{\Lambda_{i}} & I_{n_{i} \times n_{j}} \sqrt{\Lambda_{j}} \\
\sqrt{\Lambda_{j}} & I_{n_{j} \times n_{i}} \sqrt{\Lambda_{i}} & \Lambda_{j}
\end{array}\right]
$$

It is straightforward to verify that

$$
\operatorname{rank}\{S\}=\operatorname{rank}\{N\}=\max \left\{\operatorname{rank}\left\{S_{i}\right\}, \operatorname{rank}\left\{S_{j}\right\}\right\}
$$

On the other hand, the Schur complement formula yields:

$$
\begin{aligned}
& \operatorname{rank}\left\{W\left\{\mathcal{B}_{i}, \mathcal{B}_{i}\right\}\right\}=\operatorname{rank}\left\{O^{+}\right\}+\operatorname{rank}\left\{S_{i}\right\} \\
& \operatorname{rank}\left\{W\left\{\mathcal{B}_{j}, \mathcal{B}_{j}\right\}\right\}=\operatorname{rank}\left\{O^{+}\right\}+\operatorname{rank}\left\{S_{j}\right\} \\
& \operatorname{rank}\left\{W\left\{\mathcal{B}_{i} \cup \mathcal{B}_{j}, \mathcal{B}_{i} \cup \mathcal{B}_{j}\right\}\right\}=\operatorname{rank}\left\{O^{+}\right\}+\operatorname{rank}\{S\}
\end{aligned}
$$

(see [40]). Combining the above equations leads to the conclusion that the rank of $W\left\{\mathcal{B}_{i} \cup \mathcal{B}_{j}, \mathcal{B}_{i} \cup \mathcal{B}_{j}\right\}$ is upper bounded by the maximum ranks of $W\left\{\mathcal{B}_{i}, \mathcal{B}_{i}\right\}$ and $W\left\{\mathcal{B}_{j}, \mathcal{B}_{j}\right\}$. On the other hand, since $N$ is positive semidefinite, it follows from 9] that $W\left\{\mathcal{B}_{i} \cup \mathcal{B}_{j}, \mathcal{B}_{i} \cup \mathcal{B}_{j}\right\} \succeq 0$. A simple induction concludes that the output $W^{\text {opt }}$ of the matrix completion algorithm is a positive semidefinite matrix whose rank is upper bounded by $t+1$. The proof is completed by noting that $W^{\text {opt }}$ and $W^{\text {ref }}$ share the same values on their diagonals and those offdiagonal locations corresponding to the edges of the sparsity graph $\mathcal{G}$.

\section{Finite-Horizon Deterministic ODC Problem}

The primary objective of the ODC problem is to design a structurally constrained gain $K$. Assume that the matrix $K$ has $l$ free entries to be designed and all other entries are equal to zero. Denote those free entries as $h_{1}, h_{2}, \ldots, h_{l}$. The space of permissible controllers can be characterized as

$$
\mathcal{K} \triangleq\left\{\sum_{i=1}^{l} h_{i} N_{i} \mid h \in \mathbb{R}^{l}\right\}
$$

for some (fixed) matrices $N_{1}, \ldots, N_{l} \in\{0,1\}^{m \times r}$ where each of these matrices has only one nonzero element. Now, the ODC problem can be stated as follows.

\section{Finite-Horizon ODC Problem: Minimize}

$$
\sum_{\tau=0}^{p}\left(x[\tau]^{T} Q x[\tau]+u[\tau]^{T} R u[\tau]\right)+\alpha\|K\|_{F}^{2}
$$

subject to

$$
\begin{aligned}
x[0] & =c & & \\
x[\tau+1] & =A x[\tau]+B u[\tau] & & \tau=0,1, \ldots, p-1 \\
y[\tau] & =C x[\tau] & & \tau=0,1, \ldots, p \\
u[\tau] & =K y[\tau] & & \tau=0,1, \ldots, p \\
K & =h_{1} N_{1}+\ldots+h_{l} N_{l} & &
\end{aligned}
$$

over the variables $\left\{x[\tau] \in \mathbb{R}^{n}\right\}_{\tau=0}^{p},\left\{y[\tau] \in \mathbb{R}^{r}\right\}_{\tau=0}^{p},\{u[\tau] \in$ $\left.\mathbb{R}^{m}\right\}_{\tau=0}^{p}, K \in \mathbb{R}^{m \times r}$ and $h \in \mathbb{R}^{l}$. 


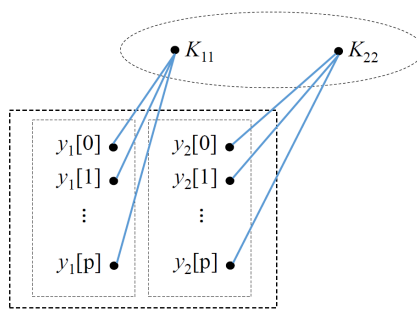

(a)

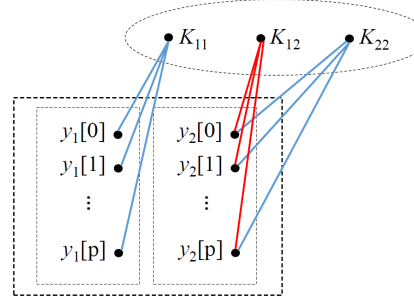

(b)
Fig. 2: Effect of a nonzero off-diagonal entry of the controller $K$ on the sparsity graph of the finite-horizon ODC: (a) a subgraph of $\mathcal{G}$ for the case where $K_{11}$ and $K_{22}$ are the only free parameters of the controller $K$, (b) a subgraph of $\mathcal{G}$ for the case where $K_{12}$ is also a free parameter of the controller.

\section{A. Sparsification of ODC Problem}

The finite-horizon ODC (11) problem is naturally a QCQP problem. Consider an arbitrary SDP relaxation of the ODC problem and let $\mathcal{G}$ be the sparsity graph of this relaxation. Due to the existence of possibly nonzero off-diagonal elements in $Q$ and $R$, certain edges (and probably cycles) may exist in the subgraphs of $\mathcal{G}$ associated with the state and input vectors $x[\tau]$ and $u[\tau]$. Under this circumstance, the treewidth of $\mathcal{G}$ could be $n$ or higher. To understand the effect of a non-diagonal controller $K$, consider the case $m=r=2$ and assume that the controller $K$ under design has three free elements as follows:

$$
K=\left[\begin{array}{cc}
K_{11} & K_{12} \\
0 & K_{22}
\end{array}\right]
$$

(i.e., $h_{1}=K_{11}, h_{2}=K_{12}$ and $h_{3}=K_{22}$ ). Figure 2 shows a part of the graph $\mathcal{G}$. It can be observed that this subgraph is acyclic for $K_{12}=0$ but has a cycle as soon as $K_{12}$ becomes a free parameter. As a result, the treewidth of $\mathcal{G}$ is contingent upon the zero pattern of $K$. In order to guarantee the existence of a low-rank solution, we diagonalize $Q, R$ and $K$ through a reformulation of the ODC problem. Note that this transformation is redundant if $Q, R$ and $K$ are already diagonal. Let $Q_{d} \in \mathbb{R}^{n \times n}$ and $R_{d} \in \mathbb{R}^{m \times m}$ be the respective eigenvector matrices of $Q$ and $R$, i.e.,

$$
Q=Q_{d}^{T} \Lambda_{Q} Q_{d}, \quad R=R_{d}^{T} \Lambda_{R} R_{d}
$$

where $\Lambda_{Q} \in \mathbb{R}^{n \times n}$ and $\Lambda_{R} \in \mathbb{R}^{m \times m}$ are diagonal matrices. Notice that there exist two constant binary matrices $\Phi_{1} \in$ $\mathbb{R}^{m \times l}$ and $\Phi_{2} \in \mathbb{R}^{l \times r}$ such that

$$
\mathcal{K}=\left\{\Phi_{1} \operatorname{diag}\{h\} \Phi_{2} \mid h \in \mathbb{R}^{l}\right\},
$$

where $\operatorname{diag}\{h\}$ denotes a diagonal matrix whose diagonal entries are inherited from the vector $h$ [41]. Now, a sparse formulation of the ODC problem can be obtained in terms of the matrices

$$
\bar{A} \triangleq Q_{d} A Q_{d}^{T}, \quad \bar{B} \triangleq Q_{d} B R_{d}^{T}, \quad \bar{C} \triangleq \Phi_{2} C Q_{d}^{T}, \quad \bar{c} \triangleq Q_{d} c,
$$

and the new set of variables $\bar{x}[\tau] \triangleq Q_{d} x[\tau], \bar{y}[\tau] \triangleq \Phi_{2} y[\tau]$ and $\bar{u}[\tau] \triangleq R_{d} u[\tau]$ for $\tau=0,1, \ldots, p$.

Reformulated Finite-Horizon ODC Problem: Minimize

$$
\sum_{\tau=0}^{p}\left(\bar{x}[\tau]^{T} \Lambda_{Q} \bar{x}[\tau]+\bar{u}[\tau]^{T} \Lambda_{R} \bar{u}[\tau]\right)+\alpha\|h\|^{2}
$$

subject to

$$
\begin{aligned}
\bar{x}[0] & =\bar{c} \times z & & \\
\bar{x}[\tau+1] & =\bar{A} \bar{x}[\tau]+\bar{B} \bar{u}[\tau] & & \tau=0,1, \ldots, p-1 \\
\bar{y}[\tau] & =\bar{C} \bar{x}[\tau] & & \tau=0,1, \ldots, p \\
z \times \bar{u}[\tau] & =R_{d} \Phi_{1} \operatorname{diag}\{h\} \bar{y}[\tau] & & =0,1, \ldots, p \\
z^{2} & =1 & &
\end{aligned}
$$

over the variables $\left\{\bar{x}[\tau] \in \mathbb{R}^{n}\right\}_{\tau=0}^{p},\left\{\bar{y}[\tau] \in \mathbb{R}^{l}\right\}_{\tau=0}^{p},\{\bar{u}[\tau] \in$ $\left.\mathbb{R}^{m}\right\}_{\tau=0}^{p}, h \in \mathbb{R}^{l}$ and $z \in \mathbb{R}$.

To cast the reformulated finite-horizon ODC as a quadratic optimization, define

$$
w \triangleq\left[\begin{array}{lllll}
z & h^{T} & \bar{x}^{T} & \bar{u}^{T} & \bar{y}^{T}
\end{array}\right]^{T} \in \mathbb{R}^{n_{w}}
$$

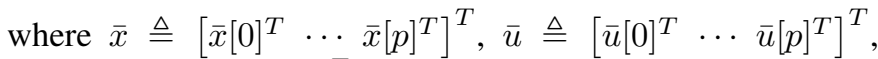

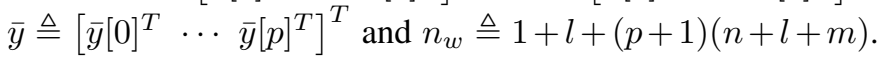
The scalar auxiliary variable $z$ plays the role of number 1 and is introduced to make the constraints $(15 \mathrm{~b}),(15 \mathrm{c}),(15 \mathrm{~d})$ and $15 \mathrm{e}$ homogenous, which will be later exploited to relax the problem (it suffices to impose the additional quadratic constraint [15f] as opposed to $z=1$ without affecting the solution).

\section{B. SDP Relaxations of ODC Problem}

In this subsection, two SDP relaxations are proposed for the reformulated finite-horizon ODC problem given in (15). For the first relaxation, there is a guarantee on the rank of the solution. In contrast, the second relaxation offers a tighter lower bound on the optimal cost of the ODC problem, but its solution might be high rank and therefore its rounding to a rank-1 solution could be more challenging.

1) Sparse SDP relaxation: Let $e_{1}, \ldots, e_{n_{w}}$ denote the standard basis for $\mathbb{R}^{n_{w}}$. The ODC problem consists of $n_{l} \triangleq$ $(p+1)(n+l)$ linear constraints given in (15b), $15 \mathrm{c})$ and (15d), which can be formulated as

$$
D^{T} w=0
$$

for some matrix $D \in \mathbb{R}^{n_{w} \times n_{l}}$. Moreover, the objective function (15a) and the constraints in (15e) and 15f) are all quadratic and can be expressed in terms of some matrices $M \in \mathbb{S}_{n_{w}},\left\{M_{i}[\tau] \in \mathbb{S}_{n_{w}}\right\}_{i=1, \ldots, m ; \tau=0,1, \ldots, p}$ and $E \triangleq e_{1} e_{1}^{T}$. This leads to the following formulation of (15).

Sparse Formulation of ODC Problem: Minimize

$$
\left\langle M, w w^{T}\right\rangle
$$

subject to

$$
\begin{aligned}
D^{T} w & =0 \\
\left\langle M_{i}[\tau], w w^{T}\right\rangle & =0 \quad i=1, \ldots, m, \quad \tau=0,1, \ldots, p \\
\left\langle E, w w^{T}\right\rangle & =1
\end{aligned}
$$

with the variable $w \in \mathbb{R}^{n_{w}}$.

For every $j=1, \ldots, n_{l}$, define

$$
D_{j}=D_{:, j} e_{j}^{T}+e_{j} D_{:, j}^{T}
$$

where $D_{:, j}$ denotes the $j$-th column of $D$. An SDP relaxation of (18) will be obtained below. 


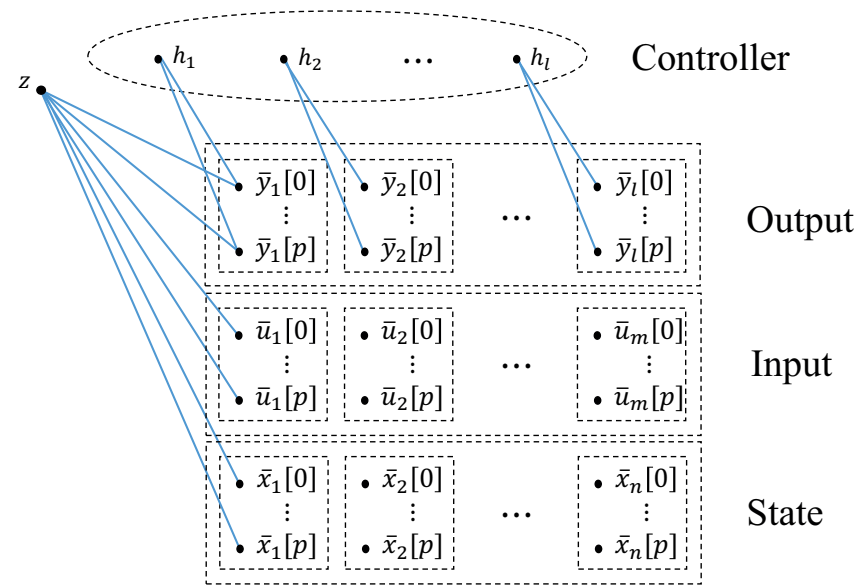

Fig. 3: Sparsity graph of the problem 20 (some edges of vertex $z$ are not shown to improve the legibility of the graph).

Sparse Relaxation of Finite-Horizon ODC: Minimize

$$
\langle M, W\rangle
$$

subject to

$$
\begin{aligned}
\left\langle D_{j}, W\right\rangle & =0 \quad j=1, \ldots, n_{l} \\
\left\langle M_{i}[\tau], W\right\rangle & =0 \quad i=1, \ldots, m, \quad \tau=0,1, \ldots, p \\
\langle E, W\rangle & =1 \\
W & \succeq 0
\end{aligned}
$$

with the variable $W \in \mathbb{S}_{n_{w}}$.

The problem 20) is a convex relaxation of the QCQP problem 18. The sparsity graph of this problem is equal to

$$
\begin{aligned}
\mathcal{G}= & \mathcal{G}\left(D_{1}\right) \cup \ldots \cup \mathcal{G}\left(D_{n_{l}}\right) \cup \mathcal{G}\left(M_{1}[0]\right) \cup \ldots \cup \mathcal{G}\left(M_{m}[0]\right) \\
& \cup \ldots \cup \mathcal{G}\left(M_{1}[p]\right) \cup \ldots \cup \mathcal{G}\left(M_{m}[p]\right),
\end{aligned}
$$

where the vertices of $\mathcal{G}$ correspond to the entries of $w$. In particular, the vertex set of $\mathcal{G}$ can be partitioned into five vertex subsets, where subset 1 consists of a single vertex associated with the variable $z$ and subsets $2-5$ correspond to the vectors $\bar{x}, \bar{u}, \bar{y}$ and $h$, respectively. The underlying sparsity graph $\mathcal{G}$ for the sparse formulation of the ODC problem is drawn in Figure 3, where each vertex of the graph is labeled by its corresponding variable. To maintain the readability of the graph, some edges of vertex $z$ are not shown in the picture. Indeed, $z$ is connected to all vertices corresponding to the elements of $\bar{x}, \bar{u}$ and $\bar{y}$ due to the linear terms in (18b).

Theorem 2. The sparsity graph of the sparse relaxation of the finite-horizon ODC problem has treewidth 2.

Proof. It follows from the graph drawn in Figure 3 that removing vertex $z$ from the sparsity graph $\mathcal{G}$ makes the remaining subgraph acyclic. This implies that the treewidth of $\mathcal{G}$ is at most 2. On the other hand, the treewidth cannot be 1 in light of the cycles of the graph.

Consider the variable $W$ of the SDP relaxation 20). The exactness of this relaxation is tantamount to the existence of an optimal rank-1 solution $W^{\text {opt }}$ for 20 . In this case, an optimal vector $w^{\text {opt }}$ for the ODC problem can be recovered by decomposing $W^{\mathrm{opt}}$ as $\left(w^{\mathrm{opt}}\right)\left(w^{\mathrm{opt}}\right)^{T}$ (note that $w$ has been defined in (16). The following observation can be made.

Corollary 1. The sparse relaxation of the finite-horizon $O D C$ problem has a matrix solution with rank at most 3.

Proof. This corollary is an immediate consequence of Theorems 1 and 2

Remark 2. Since the treewidth of the sparse relaxation of the finite-horizon $O D C$ problem (20) is equal to 2, it is possible to significantly reduce its computational complexity. More precisely, the complicating constraint $W \succeq 0$ can be replaced by positive semidefinite constraints on certain $3 \times 3$ submatrices of $W$, as given below:

$$
W\left\{\mathcal{B}_{i}, \mathcal{B}_{i}\right\} \succeq 0, \quad k=1, \ldots,|\mathcal{T}|
$$

where $\mathcal{T}$ is an optimal tree decomposition of the sparsity graph $\mathcal{G}$, and $\mathcal{B}_{1}, \ldots, \mathcal{B}_{|\mathcal{T}|}$ denote its bags. After this simplification of the hard constraint $W \succeq 0$, a quadratic number of entries of $W$ turn out to be redundant (not appearing in any constraint) and can be removed from the optimization [30], [42].

2) Dense SDP relaxation: Define $D^{\perp} \in \mathbb{R}^{n_{w} \times\left(n_{w}-n_{l}\right)}$ as an arbitrary full row rank matrix satisfying the relation $D^{T} D^{\perp}=0$. It follows from (18b that every feasible vector $w$ satisfies the equation $w=D^{\perp} \tilde{w}$, for a vector $\tilde{w} \in \mathbb{R}^{\left(n_{w}-n_{l}\right)}$. Define

$$
\begin{aligned}
\tilde{M} & =\left(D^{\perp}\right)^{T} M D^{\perp} \\
\tilde{M}_{i}[\tau] & =\left(D^{\perp}\right)^{T} M_{i}[\tau] D^{\perp} \\
\tilde{E} & =\left(D^{\perp}\right)^{T} e_{1} e_{1}^{T} D^{\perp} .
\end{aligned}
$$

The problem 18 can be cast in terms of $\tilde{w}$ as shown below.

Dense Formulation of ODC Problem: Minimize

$$
\left\langle\tilde{M}, \tilde{w} \tilde{w}^{T}\right\rangle
$$

subject to

$$
\begin{aligned}
\left\langle\tilde{M}_{i}[\tau], \tilde{w} \tilde{w}^{T}\right\rangle & =0 \quad i=1, \ldots, m, \quad \tau=0,1, \ldots, p \\
\left\langle\tilde{E}, \tilde{w} \tilde{w}^{T}\right\rangle & =1
\end{aligned}
$$

over $\tilde{w} \in \mathbb{R}^{\left(n_{w}-n_{l}\right)}$.

The SDP relaxation of the above formulation is provided next.

Dense Relaxation of Finite-Horizon ODC: Minimize

$$
\langle\tilde{M}, \tilde{W}\rangle
$$

subject to

$$
\begin{aligned}
\left\langle\tilde{M}_{i}[\tau], \tilde{W}\right\rangle & =0 \quad i=1, \ldots, m, \quad \tau=0,1, \ldots, p \\
\langle\tilde{E}, \tilde{W}\rangle & =1 \\
\tilde{W} & \succeq 0
\end{aligned}
$$

over $\tilde{W} \in \mathbb{S}_{\left(n_{w}-n_{l}\right)}$.

Remark 3. Let $\mathcal{F}_{s}$ and $\mathcal{F}_{d}$ denote the feasible sets for the sparse and dense SDP relaxation problems in (20) and (24), respectively. It can be easily seen that

$$
\left\{D^{\perp} \tilde{W}\left(D^{\perp}\right)^{T} \mid \tilde{W} \in \mathcal{F}_{d}\right\} \subseteq \mathcal{F}_{s}
$$

Therefore, the lower bound provided by the dense SDP relaxation problem (24) is equal to or tighter than that of the sparse 
SDP relaxation (20). However, the rank of the SDP solution of the dense relaxation may be high, which complicates its rounding to a rank-1 matrix. Hence, the sparse relaxation may be useful for recovering a near globally-optimal controller, while the dense relaxation may be used to bound the global optimality degree of the recovered controller.

\section{Rounding of SDP Solution to Rank-1 Matrix}

Let $W^{\text {opt }}$ either denote a low-rank solution for the sparse relaxation (20) or be equal to $D^{\perp} \tilde{W}^{\text {opt }}\left(D^{\perp}\right)^{T}$ for a lowrank solution $\tilde{W}^{\text {opt }}$ (if any) of the dense relaxation (24). If the rank of $W^{\mathrm{opt}}$ is 1 , then $W^{\mathrm{opt}}$ can be mapped back into a globally optimal controller for the ODC problem through the eigenvalue decomposition $W^{\text {opt }}=w^{\text {opt }}\left(w^{\text {opt }}\right)^{T}$. Assume that $W^{\text {opt }}$ does not have rank 1 . There are multiple heuristic methods to recover a near globally-optimal controller, some of which are delineated below.

Direct Recovery Method: If $W^{\text {opt }}$ had rank 1, then the $(2,1),(3,1), \ldots,(|h|+1,1)$ entries of $W^{\mathrm{opt}}$ would have corresponded to the vector $h^{\text {opt }}$ containing the free entries of $K^{\text {opt }}$. Inspired by this observation, if $W^{\mathrm{opt}}$ has rank greater than 1 , then a near globally-optimal controller may still be recovered from the first column of $W^{\mathrm{opt}}$. We refer to this approach as Direct Recovery Method.

Penalized SDP Relaxation: Recall that an SDP relaxation can be obtained by eliminating a rank constraint. In the case where this removal changes the solution, one strategy is to compensate for the rank constraint by incorporating an additive penalty function, denoted as $\Psi(W)$, into the objective of SDP relaxation. A common penalty function $\Psi(\cdot)$ is $\varepsilon \times \operatorname{trace}\{W\}$, where $\varepsilon$ is a design parameter. This problem is referred to as Penalized SDP Relaxation throughout this paper.

Indirect Recovery Method: Define $x$ as the aggregate state vector obtained by stacking $x[0], \ldots, x[p]$. The objective function of every proposed SDP relaxation depends strongly on $x$ and only weakly on $K$ if $\alpha$ is small. In particular, if $\alpha=0$, then the SDP objective function is not in terms of $K$. This implies that the relaxation may have two feasible matrix solutions both leading to the same optimal cost such that their first columns overlap on the part corresponding to $x$ and not the part corresponding to $h$. Hence, unlike the direct method that recovers $h$ from the first column of $W^{\text {opt }}$, it may be advantageous to first recover $x$ and then solve a second convex optimization to generate a structured controller that is able to generate state values as closely to the recovered aggregate state vector as possible. More precisely, given an SDP solution $W^{\text {opt }}$, define $\hat{x} \in \mathbb{R}^{n(p+1)}$ as a vector containing the entries $(|h|+2,1),(|h|+3,1), \ldots,(1+|h|+n(p+1), 1)$ of $W^{\text {opt }}$. Define the indirect recovery method as the convex optimization problem

$$
\begin{array}{ll}
\text { minimize } & \sum_{\tau=0}^{p}\|\hat{x}[\tau+1]-(A+B K C) \hat{x}[\tau]\|^{2} \\
\text { subject to } & K=h_{1} M_{1}+\ldots+h_{l} M_{l}
\end{array}
$$

with the variables $K \in \mathbb{R}^{m \times r}$ and $h \in \mathbb{R}^{l}$. Let $\hat{K}$ denote a solution of the above problem. In the case where $W^{\text {opt }}$ has rank 1 or the state part of the matrix $W^{\text {opt }}$ corresponds to the true solution of the ODC problem, $\hat{x}$ is the same as $x^{\text {opt }}$ and $\hat{K}$ is an optimal controller. Otherwise, $\hat{K}$ is a feasible controller that aims to make the closed-loop system follow the near-optimal state trajectory vector $\hat{x}$. As tested in Section VI, the above controller recovery method exhibits a remarkable performance on power systems.

\section{Computationally-Cheap SDP Relaxation}

Two SDP relaxations have been proposed earlier. Although these problems are convex, it may be difficult to solve them efficiently for a large-scale system. This is due to the fact that the size of each SDP matrix depends on the number of scalar variables at all times from 0 to $p$. There is an efficient approach to derive a computationally-cheap SDP relaxation. This will be explained below for the case where $r, m \leq n$.

Notation 1. For every matrix $M \in \mathbb{R}^{n_{1} \times n_{2}}$, define the sparsity pattern of $M$ as follows

$$
\mathcal{S}(M) \triangleq\left\{S \in \mathbb{R}^{n_{1} \times n_{2}} \mid \forall(i, j) M_{i j}=0 \Rightarrow S_{i j}=0\right\}
$$

With no loss of generality, we assume that $C$ has full row rank. There exists an invertible matrix $\Phi \in \mathbb{R}^{n \times n}$ such that $C \Phi=\left[\begin{array}{ll}I_{r} & 0\end{array}\right]$. Define also

$$
\mathcal{K}^{2} \triangleq\left\{\Phi_{1} S \Phi_{1}^{T} \mid S \in \mathcal{S}\left(\Phi_{2} \Phi_{2}^{T}\right)\right\} .
$$

where $\Phi_{1}$ and $\Phi_{2}$ are defined in (14). Indeed, $\mathcal{K}^{2}$ captures the sparsity pattern of the matrix $K K^{T}$. For example, if $\mathcal{K}$ consists of block-diagonal (rectangular) matrix, $\mathcal{K}^{2}$ will also include block-diagonal (square) matrices. Let $\mu \in \mathbb{R}$ be a positive number such that $Q \succ \mu \times \Phi^{-T} \Phi^{-1}$, where $\Phi^{-T}$ denotes the transpose of the inverse of $\Phi$. Define

$$
\widehat{Q}:=Q-\mu \times \Phi^{-T} \Phi^{-1} .
$$

Using the slack matrix variables

$$
X \triangleq[x[0] x[1] \ldots x[p]], \quad U \triangleq[u[0] u[1] \ldots u[p]],
$$

an efficient relaxation of the ODC problem can be obtained.

\section{Computationally-Cheap Relaxation of Finite-Horizon} ODC: Minimize

$$
\operatorname{trace}\left\{X^{T} \widehat{Q} X+\mu \mathbf{W}_{22}+U^{T} R U\right\}+\alpha \operatorname{trace}\left\{\mathbf{W}_{33}\right\}
$$

subject to

$$
\begin{aligned}
& x[\tau+1]=A x[\tau]+B u[\tau], \quad \tau=0,1, \ldots, p-1, \\
& x[0]=c,
\end{aligned}
$$

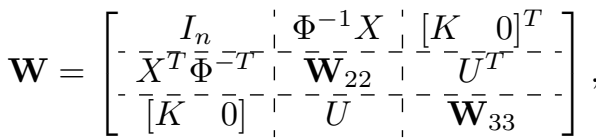

$$
\begin{aligned}
& K \in \mathcal{K} \text {, } \\
& \mathbf{W}_{33} \in \mathcal{K}^{2} \text {, } \\
& \mathbf{W} \succeq 0 \text {, }
\end{aligned}
$$

over $K \in \mathbb{R}^{m \times r}, X \in \mathbb{R}^{n \times(p+1)}, U \in \mathbb{R}^{m \times(p+1)}$ and $\mathbf{W} \in$ $\mathbb{S}_{n+m+p+1}$ (note that $\mathbf{W}_{22}$ and $\mathbf{W}_{33}$ are two blocks of the block matrix variable $\mathbf{W}$ ).

Note that the above relaxation can be naturally cast as an SDP problem by replacing each quadratic term in its objective with a new variable and then using the Schur 
complement. We refer to the SDP formulation of this problem as computationally-cheap SDP relaxation.

Theorem 3. The problem (31) is a convex relaxation of the ODC problem. Furthermore, the relaxation is exact if and only if it possesses a solution $\left(K^{o p t}, X^{o p t}, U^{o p t}, \mathbf{W}^{\text {opt }}\right)$ such that $\operatorname{rank}\left\{\mathbf{W}^{\text {opt }}\right\}=n$.

Proof. It is evident that the problem (31) is a convex program. To prove the remaining parts of the theorem, it suffices to show that the ODC problem is equivalent to (31) subject to the additional constraint $\operatorname{rank}\{\mathbf{W}\}=n$. To this end, consider a feasible solution $(K, X, U, \mathbf{W})$ such that $\operatorname{rank}\{\mathbf{W}\}=n$. Since $I_{n}$ has rank $n$, taking the Schur complement of the blocks $(1,1),(1,2),(2,1)$ and $(2,2)$ of $\mathbf{W}$ yields that

$0=\mathbf{W}_{22}-X^{T} \Phi^{-T}\left(I_{n}\right)^{-1} \Phi^{-1} X$ and $0=\mathbf{W}_{33}-K K^{T}$

On the other hand,

$$
\sum_{\tau=0}^{p}\left(x[\tau]^{T} Q x[\tau]+u[\tau]^{T} R u[\tau]\right)=\operatorname{trace}\left\{X^{T} Q X+U^{T} R U\right\}
$$

It follows from (32) and (33) that the ODC problem and its computationally cheap relaxation lead to the same objective at the respective points $(K, X, U)$ and $(K, X, U, \mathbf{W})$. In addition, it can be concluded from the Schur complement of the blocks $(1,1),(1,2),(3,1)$ and $(3,2)$ of $\mathbf{W}$ that

$$
U=\left[\begin{array}{ll}
K & 0
\end{array}\right] \Phi^{-1} X=K C X
$$

or equivalently

$$
u[\tau]=K C x[\tau] \quad \text { for } \quad \tau=0,1, \ldots, p
$$

This implies that $(K, X, U)$ is a feasible solution of the ODC problem. Hence, the optimal objective value of the ODC problem is a lower bound on that of the computationally-cheap relaxation under the additional constraint $\operatorname{rank}\{\mathbf{W}\}=n$.

Now, consider a feasible solution $(K, X, U)$ of the ODC problem. Define $\mathbf{W}_{22}=X^{T} \Phi^{-T} \Phi^{-1} X$ and $K_{2}=K K^{T}$. Observe that $\mathbf{W}$ can be written as the rank- $n$ matrix $W_{r} W_{r}^{T}$, where

$$
W_{r}=\left[\begin{array}{lll}
I_{n} & \Phi^{-1} X & {\left[\begin{array}{ll}
K & 0
\end{array}\right]^{T}}
\end{array}\right]^{T}
$$

Thus, $(K, X, U, \mathbf{W})$ is a feasible solution of the computationally-cheap SDP relaxation. This implies that the optimal objective value of the ODC problem is an upper bound on that of the computationally-cheap SDP relaxation under the additional constraint $\operatorname{rank}\{\mathbf{W}\}=n$. The proof is completed by combining this property with its opposite statement proved earlier.

The sparse and dense SDP relaxations were both obtained by defining a matrix $W$ as the product of two vectors. However, the computationally-cheap relaxation of the finite-horizon ODC Problem is obtained by defining $\mathbf{W}$ as the product of two matrices. This significantly reduces the computational complexity. To shed light on this fact, notice that the numbers of rows for the matrix variables of the sparse and dense SDP relaxations are on the order of $n p$, whereas the number of rows for the computationally-cheap SDP solution is on the order of $n+p$.
Remark 4. The computationally-cheap relaxation of the finitehorizon ODC Problem automatically acts as a penalized SDP relaxation. To explain this remarkable feature of the proposed relaxation, notice that the terms trace $\left\{\mathbf{W}_{22}\right\}$ and trace $\left\{\mathbf{W}_{33}\right\}$ in the objective function of the relaxation inherently penalize the trace of $\mathbf{W}$. This automatic penalization helps significantly with the reduction of the rank of $\mathbf{W}$ at optimality. As a result, it is expected that the quality of the relaxation will be better for higher values of $\alpha$ and $\mu$.

Remark 5. Consider the extreme case where $r=n, C=I_{n}$, $\alpha=0, p=\infty$, and the unknown controller $K$ is unstructured. This amounts to the famous $L Q R$ problem and the optimal controller can be found using the Riccati equation. It is straightforward to verify that the computationally-cheap relaxation of the ODC problem is always exact in this case even though it is infinite-dimensional. The proof is based on the following facts:

- When $K$ is unstructured, the constraint 31e and 31f] can be omitted. Therefore, there is no structural constraint on $\mathbf{W}_{33}$ and $\mathbf{W}_{31}$ (i.e., the $(3,1)$ block entry).

- Then, the constraint 31d reduces to $\mathbf{W}_{22}=$ $X^{T} \Phi^{-T} \Phi^{-1} X$ due to the term trace $\left\{\mathbf{W}_{22}\right\}$ in the objective function. Consequently, the objective function can be rearranged as $\sum_{\tau=0}^{\infty}\left(x[\tau]^{T} Q x[\tau]+u[\tau]^{T} R u[\tau]\right)$.

- The only remaining constraints are the state evolution equation and $x[0]=c$. It is known that the remaining feed-forward problem has a solution $\left(X^{\text {opt }}, U^{\text {opt }}\right)$ such that $U^{o p t}=K^{o p t} X^{o p t}$ for some matrix $K^{o p t}$.

\section{E. Stability Enforcement}

The finite-horizon ODC problem studied before had no stability conditions. We have verified in the simulations in Section VI that the closed-loop stability may be automatically guaranteed for physical systems if $p$ is large enough. In this subsection, we aim to directly enforce stability by imposing additional constraints on the proposed SDP relaxations.

Theorem 4. There exists a controller $u[\tau]=K y[\tau]$ with the structure $K \in \mathcal{K}$ to stabilize the system (1) if and only if there exist a (Lyapunov) matrix $P \in \mathbb{S}_{n}$, a matrix $K \in \mathbb{R}^{m \times r}$, and auxiliary variables $L \in \mathbb{R}^{m \times n}$ and $\mathbf{G} \in \mathbb{S}_{2 n+m}$ such that

$$
\begin{aligned}
& {\left[\begin{array}{cc}
P-I_{n} & A P+B \mathbf{G}_{32} \\
P A^{T}+\mathbf{G}_{23} B^{T} & P
\end{array}\right] \succeq 0,} \\
& K \in \mathcal{K}, \\
& \mathbf{G} \succeq 0, \\
& \mathbf{G}_{33} \in \mathcal{K}^{2}, \\
& \operatorname{rank}\{\mathbf{G}\}=n,
\end{aligned}
$$

where

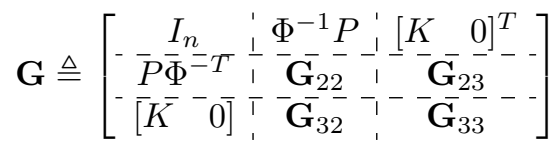

Proof. It is well-known that the system (1) is stable under a controller $u[\tau]=K y[\tau]$ if and only if there exists a positivedefinite matrix $P \in \mathbb{S}_{n}$ to satisfy the Lyapunov inequality:

$$
(A+B K C)^{T} P(A+B K C)-P+I_{n} \preceq 0
$$


or equivalently

$$
\left[\begin{array}{cc}
P-I_{n} & A P+B K C P \\
P A^{T}+P K^{T} C^{T} B^{T} & P
\end{array}\right] \succeq 0
$$

Due to the analogy between $\mathbf{W}$ and $\mathbf{G}$, the argument made in the proof of Theorem 3 can be adopted to complete the proof of this theorem (note that $\mathbf{G}_{32}$ plays the role of $K C P$ ).

Theorem 4 translates the stability of the closed-loop system into a rank- $n$ condition. Consider one of the aforementioned SDP relaxations of the ODC problem. To enforce stability, it results from Theorem 4 that two actions can be taken: (i) addition of the convex constraints 37a)-37d to SDP relaxations, (ii) compensation for the rank- $n$ condition through an appropriate convex penalization of $\mathbf{G}$ in the objective function of SDP relaxations. Note that the penalization is vital because otherwise $\mathbf{G}_{22}$ and $\mathbf{G}_{33}$ would grow unboundedly to satisfy the condition $\mathbf{G} \succeq 0$.

\section{INFINITE-HORIZON DETERMINISTIC ODC PROBLEM}

In this section, we study the infinite-horizon ODC problem, corresponding to $p=+\infty$ and subject to a stability condition.

\section{A. Lyapunov Formulation}

The finite-horizon ODC was investigated through a timedomain formulation. However, to deal with the infinite dimension of the infinite-horizon ODC and its hard stability constraint, a Lyapunov approach will be taken here. Consider the following optimization problem.

Lyapunov Formulation of ODC: Minimize

$$
c^{T} P c+\alpha\|K\|_{F}^{2}
$$

subject to

$$
\begin{aligned}
& {\left[\begin{array}{cccc}
G & G & (A G+B L)^{T} & L^{T} \\
G & Q^{-1} & 0 & 0 \\
A G+B L & 0 & G & 0 \\
L & 0 & 0 & R^{-1}
\end{array}\right] \succeq 0,} \\
& {\left[\begin{array}{cc}
P & I_{n} \\
I_{n} & G
\end{array}\right] \succeq 0,} \\
& K \in \mathcal{K} \\
& L=K C G,
\end{aligned}
$$

over $K \in \mathbb{R}^{m \times r}, L \in \mathbb{R}^{m \times n}, P \in \mathbb{S}_{n}$ and $G \in \mathbb{S}_{n}$.

It will be shown in the next theorem that the above formulation is tantamount to the infinite-horizon ODC problem.

Theorem 5. The infinite-horizon deterministic ODC problem is equivalent to finding a controller $K \in \mathcal{K}$, a symmetric Lyapunov matrix $P \in \mathbb{S}_{n}$, an auxiliary symmetric matrix $G \in \mathbb{S}_{n}$ and an auxiliary matrix $L \in \mathbb{R}^{m \times n}$ to solve the optimization problem 41).

Proof. Given an arbitrary control gain $K$, we have:

$$
\sum_{\tau=0}^{\infty}\left(x[\tau]^{T} Q x[\tau]+u[\tau]^{T} R u[\tau]\right)=x[0]^{T} P x[0]
$$

where

$$
\begin{aligned}
& P=(A+B K C)^{T} P(A+B K C)+Q+(K C)^{T} R(K C) \\
& P \succeq 0
\end{aligned}
$$

On the other hand, it is well-known that replacing the equality sign "=" in 43a with the inequality sign " $\succeq$ " does not affect the solution of the optimization problem [43]. After pre- and post-multiplying the Lyapunov inequality obtained from 43a with $P^{-1}$ and using the Schur complement formula, the constraints 43a and 43b can be combined as

$$
\left[\begin{array}{cccc}
P^{-1} & P^{-1} & S^{T} & P^{-1}(K C)^{T} \\
P^{-1} & Q^{-1} & 0 & 0 \\
S & 0 & P^{-1} & 0 \\
(K C) P^{-1} & 0 & 0 & R^{-1}
\end{array}\right] \succeq 0
$$

where $S=(A+B K C) P^{-1}$. By replacing $P^{-1}$ with a new variable $G$ in the above matrix and defining $L$ as $K C G$, the constraints (41b) and 41e will be obtained. On the other hand, according to the generalization of Schur complement in [40], the constraint 41c) holds if and only if

$$
G \succeq 0, \quad \operatorname{null}\{G\} \subseteq \operatorname{null}\left\{I_{n}\right\}, \quad \text { and } \quad P \succeq G^{-1} .
$$

Therefore, the minimization of $c^{T} P c$ subject to the constraint 41c ensures that $P=G^{-1}$ is satisfied for at least one optimal solution of the optimization problem.

Theorem 6. Consider the special case where $r=n, C=I_{n}$, $\alpha=0$ and $\mathcal{K}$ contains the set of all unstructured controllers. Then, the infinite-horizon deterministic ODC problem has the same solution as the convex optimization problem obtained from the nonlinear optimization (41) by removing its nonconvex constraint 41e.

Proof. It is easy to verify that a solution $\left(K^{\mathrm{opt}}, P^{\mathrm{opt}}, G^{\mathrm{opt}}\right.$, $L^{\text {opt }}$ ) of the convex problem stated in the theorem can be mapped to the solution $\left(L^{\mathrm{opt}}\left(G^{\mathrm{opt}}\right)^{-1}, P^{\mathrm{opt}}, G^{\mathrm{opt}}, L^{\mathrm{opt}}\right)$ of the non-convex problem (41) and vice versa (recall that $C=I_{n}$ by assumption). This completes the proof.

\section{B. SDP Relaxation}

Theorem 6 states that a classical optimal control problem can be precisely solved via a convex relaxation of the nonlinear optimization 41 by eliminating its constraint 41e. However, this simple convex relaxation does not work satisfactorily for a general control structure $K=\Phi_{1} \operatorname{diag}\{h\} \Phi_{2}$. To design a better relaxation, define

$$
w=\left[\begin{array}{lll}
1 & h^{T} & \operatorname{vec}\left\{\Phi_{2} C G\right\}^{T}
\end{array}\right]^{T}
$$

where $\operatorname{vec}\left\{\Phi_{2} C G\right\}$ is an $n l \times 1$ column vector obtained by stacking the columns of $\Phi_{2} C G$. It is possible to write every entry of the bilinear matrix term $K C G$ as a linear function of the entries of the parametric matrix $w w^{T}$. Hence, by introducing a new matrix variable $\mathbf{W}$ playing the role of $w w^{T}$, the nonlinear constraint 41e can be rewritten as a linear constraint in term of $\mathbf{W}$.

Notation 2. Define the sampling operator samp : $\mathbb{R}^{l \times n l} \rightarrow$ $\mathbb{R}^{l \times n}$ as follows:

$$
\operatorname{samp}\{X\}=\left[X_{i,(n-1) j+i}\right]_{i=1, \ldots, l ; j=1, \ldots, n} .
$$


Now, one can relax the non-convex mapping constraint $\mathbf{W}=w w^{T}$ to $\mathbf{W} \succeq 0$ and another constraint stating that the first column of $\mathbf{W}$ is equal to $w$. This yields the following convex relaxation of problem 41.

SDP Relaxation of Infinite-Horizon Deterministic ODC: Minimize

$$
c^{T} P c+\alpha \operatorname{trace}\left\{\mathbf{W}_{33}\right\}
$$

subject to

$$
\begin{aligned}
& {\left[\begin{array}{cccc}
G & G & (A G+B L)^{T} & L^{T} \\
G & Q^{-1} & 0 & 0 \\
A G+B L & 0 & G & 0 \\
L & 0 & 0 & R^{-1}
\end{array}\right] \succeq 0,} \\
& {\left[\begin{array}{ll}
P & I_{n} \\
I_{n} & G
\end{array}\right] \succeq 0,} \\
& L=\Phi_{1} \times \operatorname{samp}\left\{\mathbf{W}_{32}\right\}, \\
& \mathbf{W}=\left[\begin{array}{c:c:c}
1 & \operatorname{vec}\left\{\Phi_{2} C G\right\}^{T} & h^{T} \\
\hdashline \operatorname{vec}\left\{\Phi_{2}^{-} \bar{C} \bar{G}\right\} & \overline{\mathbf{W}}_{22}^{-} & \mathbf{W}_{23} \\
\hdashline h & \mathbf{W}_{32} & \mathbf{W}_{33}
\end{array}\right], \\
& \mathbf{W} \succeq 0,
\end{aligned}
$$

over $h \in \mathbb{R}^{l}, L \in \mathbb{R}^{m \times n}, P \in \mathbb{S}_{n}, G \in \mathbb{S}_{n}$ and $\mathbf{W} \in$ $\mathbb{S}_{1+l(n+1)}$.

If the relaxed problem (48) has the same solution as the infinite-horizon ODC in 41], the relaxation is exact.

Theorem 7. The following statements hold regarding the relaxation of the infinite-horizon deterministic ODC in (48):

i) The relaxation is exact if it has a solution $\left(h^{\text {opt }}, P^{\text {opt }}\right.$, $\left.G^{\text {opt }}, L^{\text {opt }}, \mathbf{W}^{\text {opt }}\right)$ such that $\operatorname{rank}\left\{\mathbf{W}^{\text {opt }}\right\}=1$.

ii) The relaxation always has a solution $\left(h^{\text {opt }}, P^{\text {opt }}, G^{\text {opt }}\right.$, $\left.L^{\text {opt }}, \mathbf{W}^{\text {opt }}\right)$ such that $\operatorname{rank}\left\{\mathbf{W}^{\text {opt }}\right\} \leq 3$.

Proof. Consider a sparsity graph $\mathcal{G}$ of (48), constructed as follows. The graph $\mathcal{G}$ has $1+l(n+1)$ vertices corresponding to the rows of $\mathbf{W}$. Two arbitrary disparate vertices $i, j \in\{1,2$, $\ldots, 1+l(n+1)\}$ are adjacent in $\mathcal{G}$ if $W_{i j}$ appears in at least one of the constraints of the problem (48) excluding the global constraint $\mathbf{W} \succeq 0$. For example, vertex 1 is connected to all remaining vertices of $\mathcal{G}$. The graph $\mathcal{G}$ with its vertex 1 removed is depicted in Figure 4. This graph is acyclic and therefore the treewidth of $\mathcal{G}$ is at most 2. Hence, it follows from Theorem 1 that (48) has a matrix solution with rank at most 3.

Theorem 7 states that the SDP relaxation of the infinitehorizon ODC problem has a low-rank solution. However, it does not imply that every solution of the relaxation is lowrank. Theorem 1 provides a procedure for converting a highrank solution of the SDP relaxation into a low-rank one.

\section{Computationally-Cheap Relaxation}

The aforementioned SDP relaxation has a high dimension for a large-scale system, which makes it less interesting for computational purposes. Moreover, the quality of its optimal objective value can be improved using some indirect penalty technique. The objective of this subsection is to offer a computationally-cheap SDP relaxation for the ODC problem,

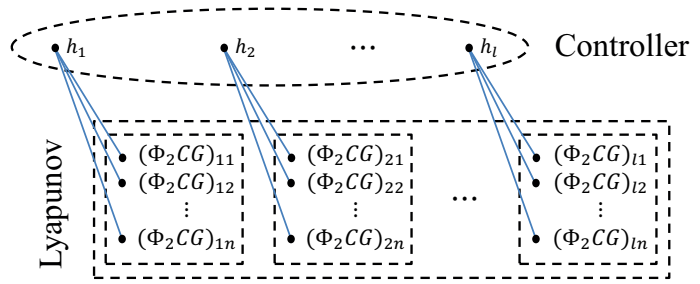

Fig. 4: The sparsity graph for the infinite-horizon deterministic ODC in the case where $\mathcal{K}$ consists of diagonal matrices (the central vertex corresponding to the constant 1 is removed for simplicity).

whose solution outperforms that of the previous SDP relaxation. For this purpose, consider again a scalar $\mu$ such that $Q \succ \mu \times \Phi^{-T} \Phi^{-1}$ and define $\hat{Q}$ according to 29 .

\section{Computationally-Cheap Relaxation of Infinite-horizon De- terministic ODC: Minimize}

$$
c^{T} P c+\alpha \operatorname{trace}\left\{\mathbf{W}_{33}\right\}
$$

subject to

$$
\begin{aligned}
& {\left[\begin{array}{cccc}
G-\mu \mathbf{W}_{22} & G & (A G+B L)^{T} & L^{T} \\
G & \widehat{Q}^{-1} & 0 & 0 \\
A G+B L & 0 & G & 0 \\
L & 0 & 0 & R^{-1}
\end{array}\right] \succeq 0} \\
& {\left[\begin{array}{ll}
P & I_{n} \\
I_{n} & G
\end{array}\right] \succeq 0}
\end{aligned}
$$

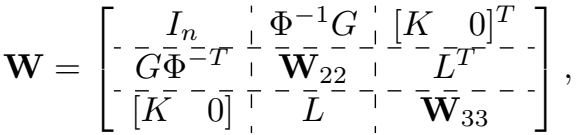

$$
\begin{aligned}
& K \in \mathcal{K} \text {, } \\
& \mathbf{W}_{33} \in \mathcal{K}^{2} \text {, } \\
& \mathbf{W} \succeq 0 \text {, }
\end{aligned}
$$

over $K \in \mathbb{R}^{m \times r}, L \in \mathbb{R}^{m \times n}, P \in \mathbb{S}_{n}, G \in \mathbb{S}_{n}$ and $\mathbf{W} \in$ $\mathbb{S}_{2 n+m}$.

The following remarks can be made regarding 49]:

- The constraint 49b corresponds to the Lyapunov inequality associated with 43a, where $\mathbf{W}_{22}$ in its first block aims to play the role of $P^{-1} \Phi^{-T} \Phi^{-1} P^{-1}$.

- The constraint (49c) ensures that the relation $P=G^{-1}$ occurs at optimality (at least for one of the solution of the problem)

- The constraint (49d) is a surrogate for the only complicating constraint of the ODC problem, i.e., $L=K C G$.

- Since no non-convex rank constraint is imposed on the problem, the rank constraint is compensated in various ways. More precisely, the entries of $\mathbf{W}$ are constrained in the objective function 49a through the term $\alpha$ trace $\left\{\mathbf{W}_{33}\right\}$, in the first block of the constraint 49b through the term $G-\mu \mathbf{W}_{22}$, and also via the constraints (49e) and (49f). These terms aim to automatically penalize the rank of $\mathbf{W}$ indirectly.

- The proposed relaxation takes advantage of the sparsity of not only $K$, but also $K K^{T}$ (through the constraint (49f)).

Theorem 8. The problem 49] is a convex relaxation of the infinite-horizon ODC problem. Furthermore, the re- 
laxation is exact if and only if it possesses a solution $\left(K^{o p t}, L^{\text {opt }}, P^{\text {opt }}, G^{\text {opt }}, \mathbf{W}^{\text {opt }}\right)$ such that $\operatorname{rank}\left\{\mathbf{W}^{\text {opt }}\right\}=n$.

Proof. The objective function and constraints of the problem (49) are all linear functions of the tuple $(K, L, P, G, \mathbf{W})$. Hence, this relaxation is indeed convex. To study the relationship between this optimization problem and the infinitehorizon ODC, consider a feasible point $(K, L, P, G)$ of the ODC formulation (41). It can be deduced from the relation $L=K C G$ that $(K, L, P, G, \mathbf{W})$ is a feasible solution of the problem (49) if the free blocks of $\mathbf{W}$ are considered as

$$
\mathbf{W}_{22}=G \Phi^{-T} \Phi^{-1} G, \quad \mathbf{W}_{33}=K K^{T}
$$

(note that 41b and (49b) are equivalent for this choice of $\mathbf{W})$. This implies that the problem (49) is a convex relaxation of the infinite-horizon ODC problem.

Consider now a solution $\left(K^{\mathrm{opt}}, L^{\mathrm{opt}}, P^{\mathrm{opt}}, G^{\mathrm{opt}}, \mathbf{W}^{\mathrm{opt}}\right)$ of the computationally-cheap SDP relaxation such that $\operatorname{rank}\left\{\mathbf{W}^{\text {opt }}\right\}=n$. Since the rank of the first block of $\mathbf{W}^{\text {opt }}$ (i.e., $I_{n}$ ) is already $n$, a Schur complement argument on the blocks $(1,1),(1,3),(2,1)$ and $(2,3)$ of $\mathbf{W}^{\text {opt }}$ yields that

$$
0=L^{\mathrm{opt}}-\left[\begin{array}{ll}
K^{\mathrm{opt}} & 0
\end{array}\right]\left(I_{n}\right)^{-1} \Phi^{-1} G^{\mathrm{opt}}
$$

or equivalently $L^{\mathrm{opt}}=K^{\mathrm{opt}} C G^{\mathrm{opt}}$, which is tantamount to the constraint (41e). This implies that $\left(K^{\mathrm{opt}}, L^{\mathrm{opt}}, P^{\mathrm{opt}}, G^{\mathrm{opt}}\right)$ is a solution of the infinite-horizon ODC problem (41) and hence the relaxation is exact. So far, we have shown that the existence of a rank- $n$ solution $\mathbf{W}^{\text {opt }}$ guarantees the exactness of the relaxation. The converse of this statement can also be proved similarly.

The variable $\mathbf{W}$ in the first SDP relaxation (48) has $1+l(n+$ 1 ) rows. In contrast, this number reduces to $2 n+m$ for the matrix $\mathbf{W}$ in the computationally-cheap relaxation 49]. This significantly reduces the computation time of the relaxation.

Corollary 2. Consider the special case where $r=n, C=I_{n}$, $\alpha=0$ and $\mathcal{K}$ contains the set of all unstructured controllers. Then, the computationally-cheap relaxation problem (49) is exact for the infinite-horizon ODC problem.

Proof. The proof follows from that of Theorem 6

\section{Controller Recovery}

In this subsection, two controller recovery methods will be described. With no loss of generality, our focus will be on the computationally-cheap relaxation problem 49.

Direct Recovery Method for Infinite-Horizon ODC: A nearoptimal controller $K$ for the infinite-horizon ODC problem is chosen to be equal to the optimal matrix $K^{\mathrm{opt}}$ obtained from the computationally-cheap relaxation problem (49).

Indirect Recovery Method for Infinite-Horizon ODC: Let $\left(K^{\mathrm{opt}}, L^{\mathrm{opt}}, P^{\mathrm{opt}}, G^{\mathrm{opt}}, \mathbf{W}^{\mathrm{opt}}\right)$ denote a solution of the computationally-cheap relaxation problem (49). Given a prespecified nonnegative number $\varepsilon$, a near-optimal controller $\hat{K}$ for the infinite-horizon ODC problem is recovered by minimizing

$$
\varepsilon \times \gamma+\alpha\|K\|_{F}^{2}
$$

subject to

$$
\left[\begin{array}{ccc}
\left(G^{\mathrm{opt}}\right)^{-1}-Q+\gamma I_{n} & (A+B K C)^{T} & (K C)^{T} \\
(A+B K C) & G^{\mathrm{opt}} & 0 \\
(K C) & 0 & R^{-1}
\end{array}\right] \succ 0
$$

$K=h_{1} N_{1}+\ldots+h_{l} N_{l}$.

over $K \in \mathbb{R}^{m \times r}, h \in \mathbb{R}^{l}$ and $\gamma \in \mathbb{R}$. Note that this is a convex program. The direct recovery method assumes that the controller $K^{\mathrm{opt}}$ obtained from the computationallycheap relaxation problem (49) is near-optimal, whereas the indirect method assumes that the controller $K^{\text {opt }}$ might be unacceptably imprecise while the inverse of the Lyapunov matrix is near-optimal. The indirect method is built on the previous SDP relaxation by fixing $G$ at its optimal value and then perturbing $Q$ as $Q-\gamma I_{n}$ to facilitate the recovery of a stabilizing controller. The underlying idea is that the SDP relaxation depends strongly on $G$ and weakly on $P$. In other words, there might be two feasible solutions with similar costs for the SDP relaxation whose $G$ parts are identical while their $P$ parts are very different. Hence, the indirect method focuses on $G$.

\section{INFINITE-HORIZON STOCHASTIC ODC PROBLEM}

This section is mainly concerned with the stochastic optimal distributed control (SODC) problem, which aims to design a stabilizing static controller $u[\tau]=K y[\tau]$ to minimize the cost function

$$
\lim _{\tau \rightarrow+\infty} \mathcal{E}\left(x[\tau]^{T} Q x[\tau]+u[\tau]^{T} R u[\tau]\right)+\alpha\|K\|_{F}^{2}
$$

subject to the system dynamics (3) and the controller requirement $K \in \mathcal{K}$, for a nonnegative scalar $\alpha$ and positive-definite matrices $Q$ and $R$. Define two covariance matrices as

$$
\Sigma_{d}=\mathcal{E}\left\{E d[0] d[0]^{T} E^{T}\right\} \quad \Sigma_{v}=\mathcal{E}\left\{F v[0] v[0]^{T} F^{T}\right\}
$$

Consider the following optimization problem.

Lyapunov Formulation of SODC: Minimize

$$
\left\langle P, \Sigma_{d}\right\rangle+\left\langle M+K^{T} R K, \Sigma_{v}\right\rangle+\alpha\|K\|_{F}^{2}
$$

subject to

$$
\begin{aligned}
& {\left[\begin{array}{cccc}
G & G & (A G+B L)^{T} & L^{T} \\
G & Q^{-1} & 0 & 0 \\
A G+B L & 0 & G & 0 \\
L & 0 & 0 & R^{-1}
\end{array}\right] \succeq 0,} \\
& {\left[\begin{array}{cc}
P & I_{n} \\
I_{n} & G
\end{array}\right] \succeq 0,} \\
& {\left[\begin{array}{cc}
M & (B K)^{T} \\
B K & G
\end{array}\right] \succeq 0,} \\
& K \in \mathcal{K} \\
& L=K C G
\end{aligned}
$$

over the controller $K \in \mathbb{R}^{m \times r}$, Lyapunov matrix $P \in \mathbb{S}_{n}$ and auxiliary matrices $G \in \mathbb{S}_{n}, L \in \mathbb{R}^{m \times n}$ and $M \in \mathbb{S}_{r}$.

Theorem 9. The infinite-horizon SODC problem adopts the non-convex formulation (55). 
Proof. It is straightforward to verify that

$$
\begin{aligned}
x[\tau] & =(A+B K C)^{\tau} x[0] \\
& +\sum_{t=0}^{\tau-1}(A+B K C)^{\tau-t-1}(E d[t]+B K F v[t])
\end{aligned}
$$

for $\tau=1,2, \ldots, \infty$. On the other hand, since the controller under design must be stabilizing, $(A+B K C)^{\tau}$ approaches zero as $\tau$ goes to $+\infty$. In light of the above equation, it can be verified that

$$
\begin{aligned}
\mathcal{E} & \left\{\lim _{\tau \rightarrow+\infty}\left(x[\tau]^{T} Q x[\tau]+u[\tau]^{T} R u[\tau]\right)\right\} \\
= & \mathcal{E}\left\{\lim _{\tau \rightarrow+\infty} x[\tau]^{T}\left(Q+C^{T} K^{T} R K C\right) x[\tau]\right\} \\
& +\mathcal{E}\left\{\lim _{\tau \rightarrow+\infty} v[\tau]^{T} F^{T} K^{T} R K F v[\tau]\right\} \\
= & \left\langle P, \Sigma_{d}\right\rangle+\left\langle(B K)^{T} P(B K)+K^{T} R K, \Sigma_{v}\right\rangle
\end{aligned}
$$

where

$$
P=\sum_{t=0}^{\infty}\left((A+B K C)^{t}\right)^{T}\left(Q+C^{T} K^{T} R K C\right)(A+B K C)^{t}
$$

Similar to the proof of Theorem 5, the above infinite series can be replaced by the expanded Lyapunov inequality (44): After replacing $P^{-1}$ and $K C P^{-1}$ in (44) with new variables $G$ and $L$, it can be concluded that:

- The condition (44) is identical to the set of constraints 55b and (55f).

- The cost function (57) can be expressed as

$$
\left\langle P, \Sigma_{d}\right\rangle+\left\langle(B K)^{T} G^{-1}(B K)+K^{T} R K, \Sigma_{v}\right\rangle+\alpha\|K\|_{F}^{2}
$$

- Since $P$ appears only once in the constraints of the optimization problem (55) (i.e., the condition (55c) ) and the objective function of this optimization includes the term $\left\langle P, \Sigma_{d}\right\rangle$, an optimal value of $P$ is equal to $G^{-1}$ (Notice that $\Sigma_{d} \succeq 0$ ).

- Similarly, the optimal value of $M$ is equal to $(B K)^{T} G^{-1}(B K)$.

The proof follows from the above observations.

The traditional $\mathrm{H}_{2}$ optimal control problem (i.e., in the centralized case) can be solved using Riccati equations. It will be shown in the next proposition that dropping the non-convex constraint (55f) results in a convex optimization that correctly solves the centralized $\mathrm{H}_{2}$ optimal control problem.

Proposition 1. Consider the special case where $r=n$, $C=I_{n}, \alpha=0, \Sigma_{v}=0$, and $\mathcal{K}$ contains the set of all unstructured controllers. Then, the SODC problem has the same solution as the convex optimization problem obtained from the nonlinear optimization [55a-55] by removing its non-convex constraint (55f).

Proof. It is similar to the proof of Theorem 6

Consider the vector $w$ defined in (46). Similar to the infinitehorizon ODC case, the bilinear matrix term $K C G$ can be represented as a linear function of the entries of the parametric matrix $\mathbf{W}$ defined as $w w^{T}$. Now, a convex relaxation can be attained by relaxing the constraint $\mathbf{W}=w w^{T}$ to $\mathbf{W} \succeq 0$ and adding another constraint stating that the first column of $\mathbf{W}$ is equal to $w$.

Relaxation of Infinite-Horizon SODC: Minimize

$$
\left\langle P, \Sigma_{d}\right\rangle+\left\langle M+K^{T} R K, \Sigma_{v}\right\rangle+\alpha \operatorname{trace}\left\{\mathbf{W}_{33}\right\}
$$

subject to

$$
\begin{aligned}
& {\left[\begin{array}{cccc}
G & G & (A G+B L)^{T} & L^{T} \\
G & Q^{-1} & 0 & 0 \\
A G+B L & 0 & G & 0 \\
L & 0 & 0 & R^{-1}
\end{array}\right] \succeq 0} \\
& {\left[\begin{array}{ll}
P & I_{n} \\
I_{n} & G
\end{array}\right] \succeq 0,} \\
& K=\Phi_{1} \operatorname{diag}\{h\} \Phi_{2}, \\
& {\left[\begin{array}{cc}
M & (B K)^{T} \\
B K & G
\end{array}\right] \succeq 0,} \\
& L=\Phi_{1} \operatorname{samp}\left\{\mathbf{W}_{32}\right\} \text {, } \\
& \mathbf{W}=\left[\begin{array}{c:c:c}
1 & \operatorname{vec}\left\{\Phi_{2} C G\right\}^{T} & h^{T} \\
\hdashline \operatorname{vec}\left\{\Phi_{2} \bar{C} \bar{G}\right\}_{1} & \mathbf{W}_{22} & \mathbf{W}_{23} \\
\hdashline h & \mathbf{W}_{32} & \mathbf{W}_{33}
\end{array}\right], \\
& \mathbf{W} \succeq 0,
\end{aligned}
$$

over the controller $K \in \mathbb{R}^{m \times r}$, Lyapunov matrix $P \in \mathbb{S}_{n}$ and auxiliary matrices $G \in \mathbb{S}_{n}, L \in \mathbb{R}^{m \times n}, M \in \mathbb{S}_{r}, h \in \mathbb{R}^{l}$ and $\mathbf{W} \in \mathbb{S}_{1+l(n+1)}$.

Theorem 10. The following statements hold regarding the convex relaxation of the infinite-horizon SODC problem:

i) The relaxation is exact if it has a solution $\left(h^{o p t}, K^{o p t}, P^{o p t}, G^{o p t}, L^{o p t}, M^{o p t}, \mathbf{W}^{\text {opt }}\right) \quad$ such that $\operatorname{rank}\left\{W^{o p t}\right\}=1$.

ii) The relaxation always has a solution $\left(h^{o p t}, K^{o p t}, P^{o p t}, G^{o p t}, L^{o p t}, M^{o p t}, \mathbf{W}^{o p t}\right) \quad$ such that $\operatorname{rank}\left\{W^{o p t}\right\} \leq 3$.

Proof. The proof is omitted (see Theorems 7 and 9).

As before, it can be deduced from Theorem 10 that the infinite-horizon SODC problem has a convex relaxation with the property that its exactness amounts to the existence of a rank-1 matrix solution $\mathbf{W}^{\text {opt }}$. Moreover, it is always guaranteed that this relaxation has a solution such that $\operatorname{rank}\left\{\mathbf{W}^{\text {opt }}\right\} \leq 3$.

A computationally-cheap SDP relaxation for the SODC problem will be derived below. Let $\mu_{1}$ and $\mu_{2}$ be two nonnegative numbers such that

$$
Q \succ \mu_{1} \times \Phi^{-T} \Phi^{-1}, \quad \Sigma_{v} \succeq \mu_{2} \times I_{r}
$$

Define $\widehat{Q}:=Q-\mu_{1} \times \Phi^{-T} \Phi^{-1}$ and $\widehat{\Sigma}_{v}:=\Sigma_{v}-\mu_{2} \times I_{r}$.

Computationally-Cheap Relaxation of Infinite-Horizon SODC: Minimize

$\left\langle P, \Sigma_{d}\right\rangle+\left\langle M, \Sigma_{v}\right\rangle+\left\langle K^{T} R K, \widehat{\Sigma}_{v}\right\rangle+\left\langle\mu_{2} R+\alpha I_{m}, \mathbf{W}_{33}\right\rangle$

subject to

$$
\left[\begin{array}{cccc}
G-\mu_{1} \mathbf{W}_{22} & G & (A G+B L)^{T} & L^{T} \\
G & \widehat{Q}^{-1} & 0 & 0 \\
A G+B L & 0 & G & 0 \\
L & 0 & 0 & R^{-1}
\end{array}\right] \succeq 0
$$




$$
\begin{aligned}
& {\left[\begin{array}{cc}
P & I_{n} \\
I_{n} & G
\end{array}\right] \succeq 0} \\
& {\left[\begin{array}{cc}
M & (B K)^{T} \\
B K & G
\end{array}\right] \succeq 0,}
\end{aligned}
$$

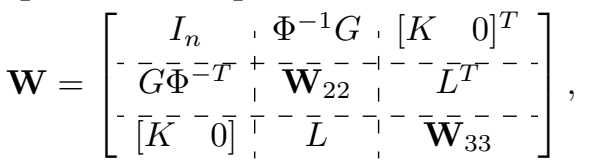

$$
\begin{aligned}
& K \in \mathcal{K} \text {, } \\
& \mathbf{W}_{33} \in \mathcal{K}^{2}, \\
& \mathbf{W} \succeq 0 \text {, }
\end{aligned}
$$

over $K \in \mathbb{R}^{m \times r}, P \in \mathbb{S}_{n}, G \in \mathbb{S}_{n}, L \in \mathbb{R}^{m \times n}, M \in \mathbb{S}_{r}$ and $\mathbf{W} \in \mathbb{S}_{2 n+m}$.

It should be noted that the constraint (60d) ensures that the relation $M=(B K)^{T} G^{-1}(B K)$ occurs at optimality.

Theorem 11. The problem 60) is a convex relaxation of the SODC problem. Furthermore, the relaxation is exact if and only if it possesses a solution $\left(K^{\text {opt }}, L^{\text {opt }}, P^{\text {opt }}, G^{\text {opt }}, M^{\text {opt }}, \mathbf{W}^{\text {opt }}\right)$ such that $\operatorname{rank}\left\{\mathbf{W}^{\text {opt }}\right\}=$ $n$.

Proof. Since the proof is similar to that of the infinite-horizon case presented earlier, it is omitted here.

For the retrieval of a near-optimal controller, the direct recovery method delineated for the infinite-horizon ODC problem can be readily deployed. However, the indirect recovery method requires some modifications, which will be explained below. Let $\left(K^{\mathrm{opt}}, L^{\mathrm{opt}}, P^{\mathrm{opt}}, G^{\mathrm{opt}}, M^{\mathrm{opt}}, \mathbf{W}^{\mathrm{opt}}\right)$ denote a solution of the computationally-cheap relaxation of SODC. A near-optimal controller $K$ for SODC may be recovered by minimizing

$$
\left\langle K^{T}\left(B^{T}\left(G^{\mathrm{opt}}\right)^{-1} B+R\right) K, \Sigma_{v}\right\rangle+\alpha\|K\|_{F}^{2}+\varepsilon \times \gamma
$$

subject to

$$
\begin{aligned}
& {\left[\begin{array}{ccc}
\left(G^{\text {opt }}\right)^{-1}-Q+\gamma I_{n} & (A+B K C)^{T} & (K C)^{T} \\
(A+B K C) & G^{\text {opt }} & 0 \\
(K C) & 0 & R^{-1}
\end{array}\right] \succ 0} \\
& K \in h_{1} N_{1}+\ldots+h_{l} N_{l} .
\end{aligned}
$$

over $K \in \mathbb{R}^{m \times r}, h \in \mathbb{R}^{l}$ and $\gamma \in \mathbb{R}$, where $\varepsilon$ is a pre-specified nonnegative number. This is a convex program.

\section{Distributed Frequency Control Problem}

Consider the distributed frequency control (DFC) problem that aims to design an optimal distributed frequency controller for a transmission system consisting of $\tilde{n}$ generator and load buses connected to each other via a physical circuit. The objective of the distributed controller is to optimally adjust the mechanical power input to each generator based on the rotor angle and frequency signals. The structure of the controller must obey a pre-determined communication topology, specifying what generators can exchange their rotor angle and frequency measurements with one another. To derive a simple state-space model of the power system, we start with the widely-used per-unit swing equation

$$
M_{i} \ddot{\theta}_{i}+D_{i} \dot{\theta}_{i}=P_{M i}-P_{E i},
$$

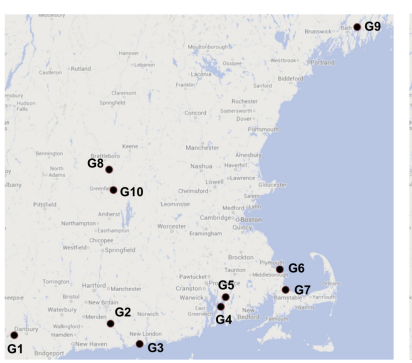

(a) Decentralized

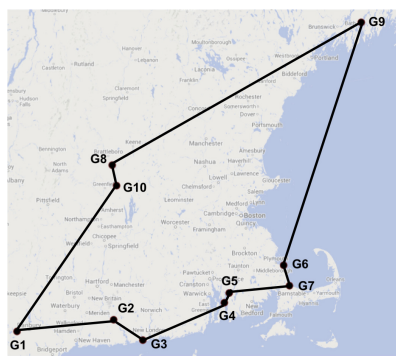

(c) Ring

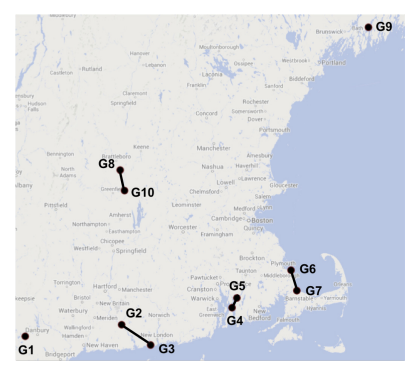

(b) Localized

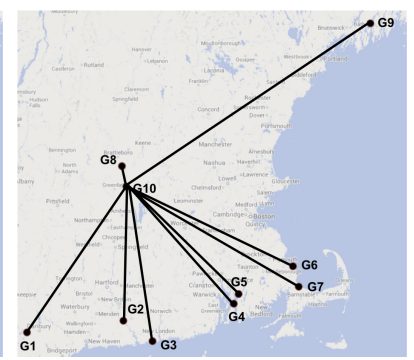

(d) Star Topology ( $G_{10}$ in center)
Fig. 5: Four communication topologies studied for IEEE 39-bus system.

where $\theta_{i}$ denotes the voltage (or rotor) angle at a generator bus $i$ (in rad), $P_{M i}$ is the mechanical power input to the generator at bus $i$ (in per unit), $P_{E i}$ is the electrical active power injection at bus $i$ (in per unit), $M_{i}$ is the inertia coefficient of the generator at bus $i$ (in $\mathrm{pu}-\mathrm{sec}^{2} / \mathrm{rad}$ ), and $D_{i}$ is the damping coefficient of the generator at bus $i$ (in pu-sec/rad) [44]. The electrical real power $P_{E i}$ in 62 comes from the nonlinear $\mathrm{AC}$ power flow equation

$P_{E i}=\sum_{j=1}^{n}\left|V_{i}\right|\left|V_{j}\right|\left[G_{i j} \cos \left(\theta_{i}-\theta_{j}\right)+B_{i j} \sin \left(\theta_{i}-\theta_{j}\right)\right]$

where $n$ denotes the number of buses in the system, $V_{i}$ is the voltage phasor at bus $i, G_{i j}$ is the line conductance, and $B_{i j}$ is the line susceptance. To simplify the formulation, a commonly-used technique is to approximate equation 63 by its corresponding DC power flow equation in which all the voltage magnitudes are assumed to be 1 per unit, each branch is modeled as a series inductor, and the angle differences across the lines are assumed to be relatively small:

$$
P_{E i}=\sum_{j=1}^{n} B_{i j}\left(\theta_{i}-\theta_{j}\right)
$$

It is possible to rewrite (64) into the matrix format $P_{E}=L \theta$, where $P_{E}$ and $\theta$ are the vectors of real power injections and voltage (or rotor) angles at only the generator buses (after removing the load buses and the intermediate zero buses). In this equation, $L$ denotes the Laplacian matrix and can be found as follows [45]:

$$
L_{i i}=\sum_{k=1, k \neq i}^{\bar{n}} B_{i k}^{\mathrm{Kron}} \text { and } L_{i j}=-B_{i j}^{\mathrm{Kron}}(i \neq j)
$$

where $B^{\mathrm{Kron}}$ is the susceptance of the Kron reduced admittance matrix $Y^{\text {Kron }}$ defined as

$Y_{i j}^{\mathrm{Kron}}=Y_{i j}-Y_{i k} Y_{k j} / Y_{k k} \quad(i, j=1,2, \ldots, n$ and $i, j \neq k)$

where $k$ is the index of the non-generator bus to be eliminated from the admittance matrix and $\bar{n}$ is the number of generator 


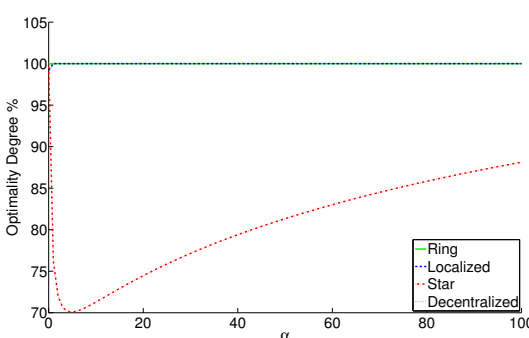

(a) Optimality degree for finite-horizon DFC

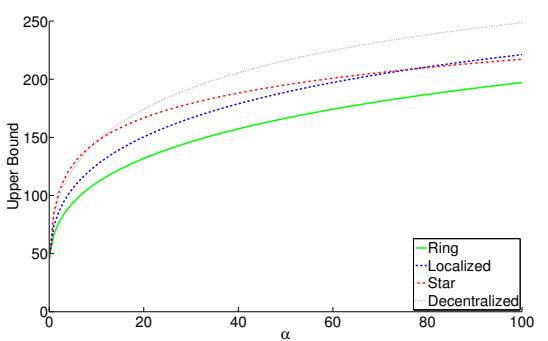

(b) Near-optimal cost for finite-horizon DFC

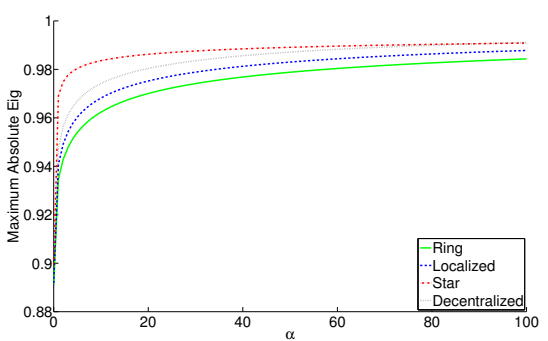

(c) Stability degree for finite-horizon DFC

Fig. 6: A near-optimal controller $\hat{K}$ is designed to solve the finite-horizon DFC problem for every control topology given in Figure 5 and every $\alpha$ between 0 and 100: (a) optimality degree, (b) near-optimal cost, and (c) closed-loop stability (maximum of the absolute eigenvalues of the closed-loop system).

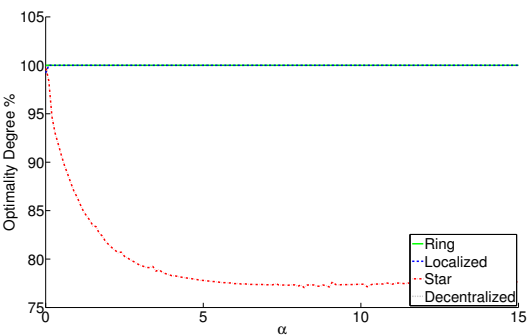

(a) Optimality degree for infinite-horizon DFC

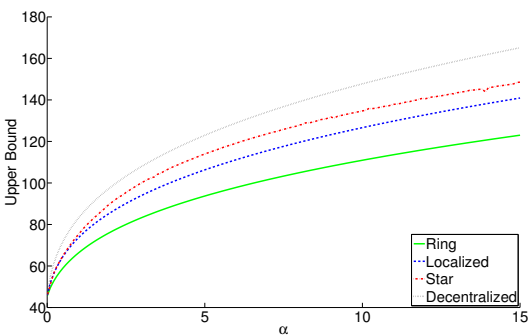

(b) Near-optimal cost for infinite-horizon DFC

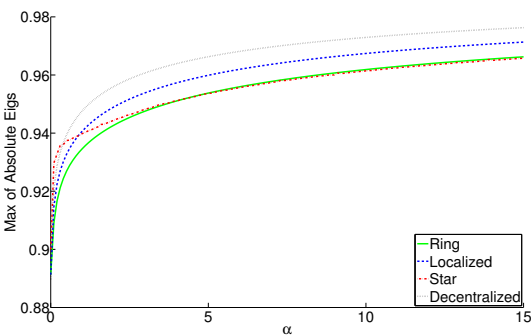

(c) Stability degree for infinite-horizon DFC

Fig. 7: A near-optimal controller $\hat{K}$ is designed to solve the infinite-horizon DFC problem for every control topology given in Figure 5 and every $\alpha$ between 0 and 15: (a) optimality degree, (b) near-optimal cost, and (c) closed-loop stability (maximum of the absolute eigenvalues of the closed-loop system).

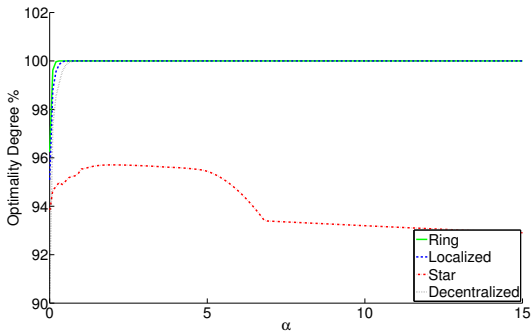

(a) Optimality degree for stochastic DFC

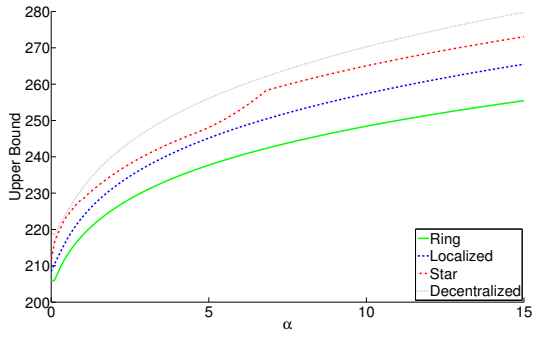

(b) Near-optimal cost for stochastic DFC

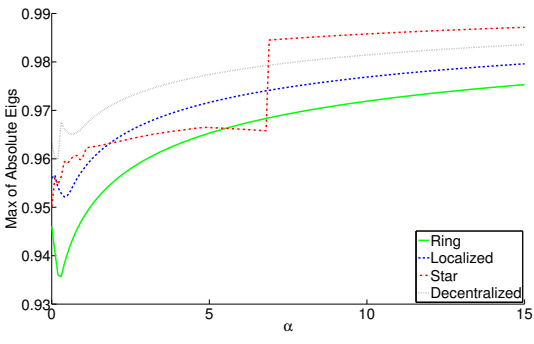

(c) Stability degree for stochastic DFC

Fig. 8: A near-optimal controller $\hat{K}$ is designed to solve the stochastic DFC problem for every control topology given in Figure 5 and every $\alpha$ between 0 and 15 under the assumptions that $\Sigma_{d}=I$ and $\Sigma_{v}=0$ : (a) optimality degree, (b) near-optimal cost, and (c) closed-loop stability

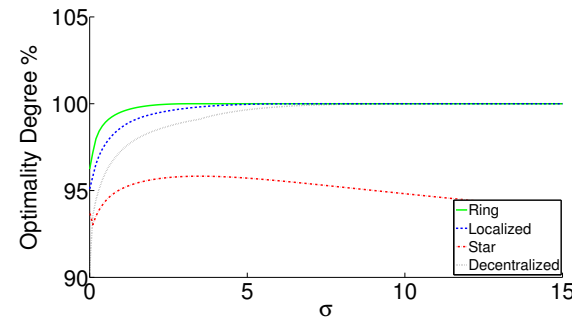

(a) Optimality degree for stochastic DFC

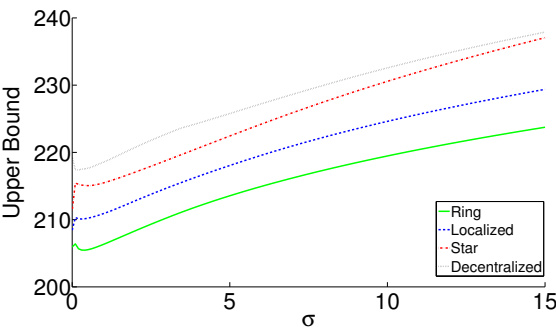

(b) Near-optimal cost for stochastic DFC

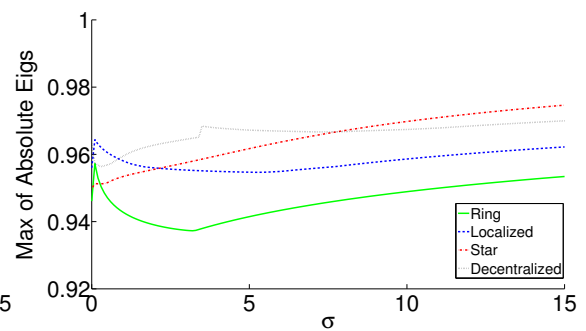

(c) Stability degree for stochastic DFC

Fig. 9: A near-optimal controller $\hat{K}$ is designed to solve the stochastic DFC problem for every control topology given in Figure 5 and every $\sigma$ between 0 and 15 under the assumptions that $\Sigma_{d}=I, \alpha=0$ and $\Sigma_{v}=\sigma I$ : (a) optimality degree, (b) near-optimal cost, and (c) closed-loop stability

buses. Note that the Kron reduction method aims to eliminate the static buses of the network because the dynamics and interactions of only the generator buses are of interest [46].

By defining the rotor angle state vector as $\theta=\left[\theta_{1}, \ldots, \theta_{\bar{n}}\right]^{T}$ and the frequency state vector as $w=\left[w_{1}, \ldots, w_{\bar{n}}\right]^{T}$ and by substituting the matrix format of $P_{E}$ into 62, the state-space model of the swing equation used for frequency control in power systems could be written as

$\left[\begin{array}{c}\dot{\theta} \\ \dot{w}\end{array}\right]=\left[\begin{array}{cc}0_{\bar{n} \times \bar{n}} & I_{\bar{n}} \\ -M^{-1} L & -M^{-1} D\end{array}\right]\left[\begin{array}{c}\theta \\ w\end{array}\right]+\left[\begin{array}{c}0_{\bar{n} \times \bar{n}} \\ M^{-1}\end{array}\right] P_{M}, \quad y=\left[\begin{array}{c}\theta \\ w\end{array}\right]$

where $M=\operatorname{diag}\left(M_{1}, \ldots, M_{\bar{n}}\right)$ and $D=\operatorname{diag}\left(D_{1}, \ldots, D_{\bar{n}}\right)$.
It is assumed that both rotor angle and frequency are available for measurement at each generator (implying that $C=I_{2 \bar{n}}$ ).

We evaluate the performance of the aforementioned SDP relaxations on the problem of designing an optimal distributed frequency control for IEEE 39-Bus New England Power System. By substituting the per-unit inertia (M) and damping (D) coefficients for the 10 generators of IEEE 39-Bus system, the continuous-time state space model matrices $A_{c}, B_{c}$ and $C_{c}$ can be found [47]. The system is then discretized to the discrete-time model matrices $A, B$ and $C$ with the sampling time of 0.2 second. The initial values of the rotor angle $\left(\theta_{0}\right)$ 
were calculated by solving power (or load) flow problem for the system using MATPOWER [48]. In practice, the rotor speed does not vary significantly from synchronous speed and thus the initial frequency $\left(w_{0}\right)$ was assumed to be 1.0 per unit.

We aim to solve thousands of SDP relaxations for these systems and evaluate their performance for different control topologies and a wide range of values for $\left(\alpha, \Sigma_{d}, \Sigma_{v}\right)$. Note that the computation time for each SDP relaxation is from a fraction of a second to 4 seconds on a desktop computer with an Intel Core i7 quad-core $3.4 \mathrm{GHz}$ CPU and $16 \mathrm{~GB}$ RAM.

The 39-bus system has 10 generators, labeled as $G_{1}, G_{2}, \ldots, G_{10}$. Four communication topologies are considered in this work: decentralized, localized, star, and ring. These topologies are visualized in Figure 5 The locations of the generators in the figure are based on the exact coordinates of the power plants named in [49]. Note that $G_{1}$ represents a group of generators, but it is considered as a single node near the border between New York and Connecticut in this map. $G_{4}$ and $G_{5}$ are very close in distance, but $G_{4}$ was somewhat shifted from its real coordinates to make the communication link between them visible in this map.

Define the optimality degree associated with a recovered controller as:

$$
\text { Optimality degree }(\%)=100-\frac{\text { upper bound }- \text { lower bound }}{\text { upper bound }} \times 100
$$

where "upper bound" and "lower bound" denote the cost of the recovered near globally-optimal controller and the optimal objective of SDP relaxation, respectively. Notice that the employed optimality measure evaluates the performance within the specified set of controllers. For example, the optimality degree of $100 \%$ means that among the linear static controllers possessing the given structure, a globally optimal one is obtained.

\section{A. Finite-Horizon DFC}

Assume that $Q=I, R=0.1 I$, and $p=80$. Suppose also that $\alpha$ is a parameter between 0 and 100. The goal is to solve a finite-horizon DFC problem for each value of $\alpha$ and for each of the four aforementioned communication topologies. This will be achieved in two steps. First, the convex relaxation of the finite-horizon DFC is solved. Second, a near-optimal controller $\hat{K}$ is designed by choosing the best solution of the direct and indirect recovery methods. The results are reported in Figures 6(a)-(c). The following observations can be made:

- The designed controllers are almost $100 \%$ globally optimal for three control topologies of decentralized, localized and ring, and this result holds for all possible values of $\alpha$. The optimality degree for the star controller is above $70 \%$ and approaches $100 \%$ (even though slowly) as $\alpha$ grows.

- For every value of $\alpha \in[0,100]$, the decentralized controller has the lowest performance, while the ring controller offers the best performance.

- The closed-loop system is always stable for all 4 control topologies and all possible values of $\alpha$.

\section{B. Infinite-Horizon DFC}

Consider the problem of solving an infinite-horizon DFC problem for each value of $\alpha$ in the interval $[0,15]$ and each of the four aforementioned communication topologies. Similar to the previous experiment, stabilizing near-optimal controllers are designed for all of these cases. The results are summarized in Figure 7

\section{Stochastic DFC}

Assume that $\Sigma_{d}$ is equal to $I$. We consider two different scenarios:

i) Suppose that $\Sigma_{v}=0$, while $\alpha$ varies from 0 to 15 . We solve the convex relaxation of each stochastic DFC, from which a near-optimal solution $\hat{K}$ is designed by choosing the best solution of the direct and indirect recovery methods. The outcome is plotted in Figure 8.

ii) Suppose that $\alpha=0$, while $\Sigma_{v}$ is equal to $\sigma I$ with $\sigma$ varying between 0 and 15 . As before, we design a nearoptimal controller for each stochastic DFC problem. The results are reported in Figure 9

\section{Performance Evaluation}

In the above experiments, three convex relaxations were solved for the finite-horizon DFC, infinite-horizon DFC and stochastic DFC problems. Interestingly, the designed controllers were all stabilizing (with no exception), and their optimality degrees were close to $99 \%$ in case of the decentralized, localized and ring structures. In case of the star structure, the optimality degree was higher than $70 \%$ in finite-horizon DFC, higher than $77 \%$ in infinite-horizon DFC and around $94 \%$ for various levels of $\sigma$ and $\alpha$ in stochastic DFC.

\section{CONCLUSIONS}

This paper studies the optimal distributed control (ODC) problem for linear discrete-time deterministic and stochastic systems. The objective is to design a static distributed controller with a pre-determined structure to minimize a quadratic cost functional. We propose semidefinite programming (SDP) relaxations for both time-domain and Lyapunovdomain formulations of the ODC problem. The notion of tree decomposition is exploited to prove the existence of a low-rank solution for the SDP relaxation problems with rank at most 3. Computationally-cheap relaxations are also developed for finite-horizon, infinite-horizon, and stochastic ODC problems. These relaxations are guaranteed to exactly solve the LQR and $H_{2}$ problems for the classical centralized control problem. The proposed relaxation techniques are tested on the problem of the distributed frequency control of power systems, leading to near-optimal controllers with global optimality degrees above $99 \%$. The generalization of the results of this paper to dynamics controllers is left as future work.

\section{REFERENCES}

[1] H. S. Witsenhausen, "A counterexample in stochastic optimum control," SIAM Journal of Control, vol. 6, no. 1, 1968.

[2] J. N. Tsitsiklis and M. Athans, "On the complexity of decentralized decision making and detection problems," Conference on Decision and Control, 1984.

[3] R. D'Andrea and G. Dullerud, "Distributed control design for spatially interconnected systems," IEEE Transactions on Automatic Control, vol. 48, no. 9, pp. 1478-1495, 2003.

[4] B. Bamieh, F. Paganini, and M. A. Dahleh, "Distributed control of spatially invariant systems," IEEE Transactions on Automatic Control, vol. 47, no. 7, pp. 1091-1107, 2002. 
[5] C. Langbort, R. Chandra, and R. D'Andrea, "Distributed control design for systems interconnected over an arbitrary graph," IEEE Transactions on Automatic Control, vol. 49, no. 9, pp. 1502-1519, 2004.

[6] N. Motee and A. Jadbabaie, "Optimal control of spatially distributed systems," IEEE Transactions on Automatic Control, vol. 53, no. 7, pp. $1616-1629,2008$.

[7] G. Dullerud and R. D'Andrea, "Distributed control of heterogeneous systems," IEEE Transactions on Automatic Control, vol. 49, no. 12, pp. 2113-2128, 2004

[8] T. Keviczky, F. Borrelli, and G. J. Balas, "Decentralized receding horizon control for large scale dynamically decoupled systems," Automatica, vol. 42 , no. 12 , pp. 2105-2115, 2006.

[9] F. Borrelli and T. Keviczky, "Distributed LQR design for identical dynamically decoupled systems," IEEE Transactions on Automatic Control, vol. 53, no. 8, pp. 1901-1912, 2008.

[10] D. D. Siljak, "Decentralized control and computations: status and prospects," Annual Reviews in Control, vol. 20, pp. 131-141, 1996.

[11] J. Lavaei, "Decentralized implementation of centralized controllers for interconnected systems," IEEE Transactions on Automatic Control, vol. 57, no. 7, pp. 1860-1865, 2012.

[12] M. Fardad, F. Lin, and M. R. Jovanović, "On the optimal design of structured feedback gains for interconnected systems," in 48th IEEE Conference on Decision and Control, 2009, pp. 978-983.

[13] F. Lin, M. Fardad, and M. R. Jovanović, "Augmented Lagrangian approach to design of structured optimal state feedback gains," IEEE Transactions on Automatic Control, vol. 56, no. 12, pp. 2923-2929, 2011.

[14] J. Geromel, J. Bernussou, and P. Peres, "Decentralized control through parameter space optimization," Automatica, vol. 30, no. 10, pp. 1565 1578, 1994.

[15] R. A. Date and J. H. Chow, "A parametrization approach to optimal $\mathrm{H}_{2}$ and $H_{\infty}$ decentralized control problems," Automatica, vol. 29, no. 2, pp. $457-463,1993$.

[16] G. Scorletti and G. Duc, "An LMI approach to dencentralized $H_{\infty}$ control," International Journal of Control, vol. 74, no. 3, pp. 211-224, 2001.

[17] G. Zhai, M. Ikeda, and Y. Fujisaki, "Decentralized $H_{\infty}$ controller design: a matrix inequality approach using a homotopy method," $\mathrm{Au}$ tomatica, vol. 37, no. 4, pp. $565-572,2001$.

[18] G. A. de Castro and F. Paganini, "Convex synthesis of localized controllers for spatially invariant systems," Automatica, vol. 38, no. 3, pp. 445 - 456, 2002.

[19] B. Bamieh and P. G. Voulgaris, "A convex characterization of distributed control problems in spatially invariant systems with communication constraints," Systems \& Control Letters, vol. 54, no. 6, pp. 575 - 583, 2005.

[20] X. Qi, M. Salapaka, P. Voulgaris, and M. Khammash, "Structured optimal and robust control with multiple criteria: a convex solution," IEEE Transactions on Automatic Control, vol. 49, no. 10, pp. 1623$1640,2004$.

[21] N. Matni and J. C. Doyle, "A dual problem in $H_{2}$ decentralized control subject to delays," American Control Conference, 2013.

[22] M. Rotkowitz and S. Lall, "A characterization of convex problems in decentralized control," IEEE Transactions on Automatic Control, vol. 51, no. 2, pp. 274-286, 2006.

[23] P. Shah and P. Parrilo, " $\mathrm{H}_{2}$-optimal decentralized control over posets: A state-space solution for state-feedback," IEEE Transactions on Automatic Control,, vol. 58, no. 12, pp. 3084-3096, Dec 2013.

[24] L. Lessard and S. Lall, "Optimal controller synthesis for the decentralized two-player problem with output feedback," American Control Conference, 2012.

[25] A. Lamperski and J. C. Doyle, "Output feedback $\mathrm{H}_{2}$ model matching for decentralized systems with delays," American Control Conference, 2013.

[26] M. Rotkowitz and N. Martins, "On the nearest quadratically invariant information constraint," IEEE Transactions on Automatic Control, vol. 57, no. 5, pp. 1314-1319, 2012.

[27] T. Tanaka and C. Langbort, "The bounded real lemma for internally positive systems and H-infinity structured static state feedback," IEEE Transactions on Automatic Control, vol. 56, no. 9, pp. 2218-2223, 2011.

[28] A. Rantzer, "Distributed control of positive systems," http://arxiv.org/ abs/1203.0047. 2012.

[29] G. Pataki, "On the rank of extreme matrices in semidefinite programs and the multiplicity of optimal eigenvalues," Mathematics of Operations Research, vol. 23, pp. 339-358, 1998.
[30] R. Madani, G. Fazelnia, S. Sojoudi, and J. Lavaei, "Low-rank solutions of matrix inequalities with applications to polynomial optimization and matrix completion problems," Conference on Decision and Control, 2014.

[31] J. F. Sturm and S. Zhang, "On cones of nonnegative quadratic functions," Mathematics of Operations Research, vol. 28, pp. 246-267, 2003.

[32] J. Lavaei and S. H. Low, "Zero duality gap in optimal power flow problem," IEEE Transactions on Power Systems, vol. 27, no. 1, pp. 92-107, 2012

[33] S. Sojoudi and J. Lavaei, "Physics of power networks makes hard optimization problems easy to solve," IEEE Power \& Energy Society General Meeting, 2012.

[34] S. Sojoudi and J. Lavaei, "Exactness of semidefinite relaxations for nonlinear optimization problems with underlying graph structure," SIAM Journal on Optimization, vol. 24, pp. 1746-1778, 2015.

[35] S. Sojoudi and J. Lavaei, "On the exactness of semidefinite relaxation for nonlinear optimization over graphs: Part I," IEEE Conference on Decision and Control, 2013.

[36] S. Sojoudi and J. Lavaei, "On the exactness of semidefinite relaxation for nonlinear optimization over graphs: Part II," IEEE Conference on Decision and Control, 2013.

[37] D. Bienstock and G. Munoz, "LP formulations for mixed-integer polynomial optimization problems," 2015.

[38] J. Lavaei, "Optimal decentralized control problem as a rank-constrained optimization," Allerton, 2013.

[39] H. L. Bodlaender, "A tourist guide through treewidth," Acta cybernetica, vol. 11, no. 1-2, p. 1, 1994.

[40] D. Carlson, E. Haynsworth, and T. Markham, "A generalization of the Schur complement by means of the Moore-Penrose inverse," SIAM Journal on Applied Mathematics, vol. 26, no. 1, pp. 169-175, 1974.

[41] J. Lavaei and A. G. Aghdam, "Control of continuous-time LTI systems by means of structurally constrained controllers," Automatica, vol. 44, no. $1,2008$.

[42] M. Fukuda, M. Kojima, K. Murota, and K. Nakata, "Exploiting sparsity in semidefinite programming via matrix completion I: general framework," SIAM J. Optimization, 2000.

[43] S. Boyd and L. Vandenberghe, Convex Optimization. Cambridge, 2004.

[44] M. A. Pai, Energy Function Analysis for Power System Stability. Kluwer Academic Publishers, Boston, 1989.

[45] F. Dorfler and F. Bullo, "Novel insights into lossless AC and DC power flow," IEEE Power and Energy Society General Meeting, 2013.

[46] A. R. Bergen and V. Vittal, Power Systems Analysis. Prentice Hall, 1999, vol. 2.

[47] I. E. Atawi, "An advance distributed control design for wide-area power system stability," Ph.D. dissertation, Swanson School Of Engineering, University of Pittsburgh, Pittsburgh,Pennsylvania, 2013.

[48] R. Zimmerman, C. Murillo-Sanchez, and R. Thomas, "Matpower: Steady-state operations, planning, and analysis tools for power systems research and education," IEEE Transactions on Power Systems, vol. 26, no. 1, pp. 12-19, Feb 2011.

[49] M. Ilic, J. Lacalle-Melero, F. Nishimura, W. Schenler, D. Shirmohammadi, A. Crough, and A. Catelli, "Short-term economic energy management in a competitive utility environment," IEEE Transactions on Power Systems, vol. 8, no. 1, pp. 198-206, Feb 1993.

Ghazal Fazelnia is a Ph.D. student in Electrical Engineering at Columbia University. She is a Bronze Medalist in National Mathematics Olympiad, Iran in 2008 and recipient of Microsoft Research Graduate Womens Scholarship in 2015 .

Ramtin Madani received the Ph.D. degree in Electrical Engineering from Columbia University in 2015. He is currently a Postdoctoral Scholar in the Department of Industrial Engineering and Operations Research at University of California, Berkeley.

Abdulrahman Kalbat is an Assistant Professor in the Electrical Engineering Department at United Arab Emirates University. He received the Ph.D. degree in Electrical Engineering from Columbia University in 2015.

Javad Lavaei is an Assistant Professor in the Department of Industrial Engineering and Operations Research at University of California, Berkeley. He obtained his Ph.D. degree in Computing and Mathematical Sciences from California Institute of Technology in 2011. He has won multiple awards, including NSF CAREER Award, Office of Naval Research Young Investigator Award, DARPA Young Faculty Award, Google Faculty Research Award, Donald P. Eckman Award, Resonate Award, and INFORMS Optimization Society Prize for Young Researchers. 